This is a work of the United States Government. In accordance with 17 U.S.C. 105, no copyright protection is available for such works under U.S. Law. Access to this work was provided by the University of Maryland, Baltimore County (UMBC) ScholarWorks@UMBC digital repository on the Maryland Shared Open Access (MD-SOAR) platform.

Please provide feedback

Please support the ScholarWorks@UMBC repository by emailing scholarworks-group@umbc.edu and telling us what having access to this work means to you and why it's important to you. Thank you. 


\title{
A Graph-based Automatic Services Composition based on Cost Estimation Heuristic
}

\author{
Yunsu Lee ${ }^{1}$, Boonserm Kulvatunyou ${ }^{1}$, Minchul Lee ${ }^{1}$, Yun Peng ${ }^{2}$, Nenad Ivezic ${ }^{1}$ \\ ${ }^{1}$ Systems Integration Division, National Institute of Standards and Technology \\ ${ }^{2}$ Computer Science and Electrical Engineering, University of Maryland, Baltimore County
}

\begin{abstract}
Currently, both software and hardware are being virtualized and offered as services on the internet. Companies have an opportunity to improve their workflows by composing services that best suit their quality and cost requirements. However, as more services become available, computer-aided services discovery and composition become essential. Traditional service representation and planning algorithms do not adequately address non-functional characteristics, large numbers of similar operators (i.e., services), and limited numbers of objects (i.e., inputs and outputs per service). This paper analyzes existing work in automatic services composition, service representation and planning algorithms and proposes a new framework to address those needs. It proves that the proposed framework provides an admissible heuristic based on cost estimations that guarantee a minimum cost solution, if one exists.
\end{abstract}

\section{Introduction}

Software and hardware components are trending toward modular atomic services (rather than large monolithic applications) on the cloud. This trend enables companies to improve their operations by choosing best-of-breed services for specific functions. For example, a food manufacturer can compose their production planning using its in-house information gathering services and external best-of-breed recipe reformulation services based on ingredient characteristics of a given batch. Companies can also quickly respond to disruptions and the customer's changing needs by composing services to meet new requirements. Cloud platforms or marketplaces such as the Smart Manufacturing Platform proposed by the Smart Manufacturing Leadership Coalition (SMLC) [12] facilitate this trend. The open access of such marketplaces means that customers may encounter a large number of available services.

In such situation, computer-aided services composition is essential. For example, a user can write a rule to detect an event from a Customer Relationship Management (CRM) service which then triggers an email service to send the event details to a particular address. Despite such simple services composition structure, services that composed together need to be functionally and nonfunctionally compatible. For that, today's existing tools provide only a simple categorization of available services. Therefore, it is a challenge for the user to manually determine the functional and non-functional compatibility between services. In an enterprise business environment, the composition task is typically more complex than the simple email trigger example above. Therefore, a computational aid is essential for making services search and composition more efficient and effective in such an open marketplace for service offerings. 
There are two primary issues in developing a computational aid for services search and composition. First, currently available computer interpretable service descriptions are insufficient. Typically, services have functional and non-functional characteristics. Currently, there are several existing standards and efforts to describe the characteristics of services. WSDL (Web Services Description Language) [3] and Open API [4] are the standards used by many software vendors. However, these standards focus more on the non-functional characteristics such as the transfer protocol specification that are essential for ensuring the secure and reliable transmission of information between different services. The standards offer no more than inputs and outputs of the service and their formats in term of the functional characteristics. Those input and output formats provide insufficient information for the automated composability analysis that supports finding and evaluating of combined components to perform a desired task. The other issue is that there is no automatic computational method that is best suitable for the services composition problem.

In existing automatic services composition works such as in [5-7], services were represented as operators and user's requirements were represented as the initial and the goal conditions. Then, the services composition problem was formulated as a classical planning problem to find a set of services (operators) that can transition the initial condition to the goal condition while satisfying input/output and pre/post-conditions of the services. However, Artificial Intelligent (AI) planning methods for solving classical planning problem have a few drawbacks when applying them to the services composition problem.

First, these methods are typically applicable to a problem with a small number of possible operators (e.g., moving blocks) and a large number of objects (e.g., hundreds of blocks). On the other hand, services composition problem typically deals with a large number of operators (huge number of services on the cloud), and a limited number of objects (e.g., getting a flight, renting a car, and registering a hotel, transforming a piece of information or a workpiece). Second, AI planning methods do not deal with the situation where there are a large number of same or similar services (e.g., hundreds of travel agent services, tens of possible scheduling algorithms, or manufacturing processes). Lastly, AI planning methods are typically computationally expensive due to the embedded Sussman anomaly avoidance [8-9]. For services composition problems, however, interleaving conditions between sub-goals in Sussman anomaly can be detected and prevented a priori.

The main objective of this research is to develop a computer-aided services search and composition framework for an open cloud service marketplace environment. To realize such a framework, this paper presents a method for representing a service and a novel graph-based algorithm using a cost estimation heuristic for services search and composition. A proof is provided that the heuristic guarantees the minimal cost solution subgraph if one exists. Finally, the results of an experimental comparison with other algorithms are presented. They indicate the exceptional performance and scalability of the algorithm.

\section{Related Work}

In this section, works closely relevant to our research are presented. First, we review existing specifications for service descriptions. Second, we dive into the detail of functional representation within the service description. Third, a state-of-the-art review on existing automatic services composition methods is provided. Two major means to automatic service search and composition methods are investigated, AI planning-based and graph search-based. 


\subsection{Service description method}

The rise of Internet computing resulted in Web-based interface definition languages. W3C (World Wide Web Consortium) WSDL [3] is a predominant one. Basic semantics and structure of WSDL are similar to that of programming language APIs (Application Programming Interfaces). More recently, a service description specification called Open API [4] has been introduced. A WSDL and Open API function, however, does not necessarily tie to a source code function - allowing it to represent the aggregate, business- or user-oriented functionality. WSDL also allows for rich description via XML (eXtensible Markup Language) Schema specification of the input and output and via structured annotation on any information element. However, WSDL does not standardize any semantics of the annotation. The strength of WSDL is in the standardized semantics of its transfer protocol specification that is essential for measuring non-functional compatibility.

Several efforts have been proposed to enhance how Web services descriptions describe function. Prior work in this area include OWL-S (Web Ontology Language for Ser-vices) [10], SAWSDL (Semantic Annotations for WSDL and XML Schema) [11], and WSMO (Web Service Modeling Ontology) [12]. SAWSDL enhances WSDL and associated XML Schema semantics by adding attributes to WSDL entities that point to concepts in a semantically rich ontology. SAWSDL does not define any additional semantics to describe functionalities; it only provides a mechanism to link to the referenced semantics.

OWL-S (Semantic Markup for Web Services) and WSMO (Web Services Modeling Ontology) are similar in their efforts to define ontologies for service descriptions (called Service Profile in OWL-S and Capability in WSMO). Both of them rely on a similar set of elements that describe 1) pre- and post-conditions associated with information used and produced by a service and 2) preand post-conditions associated with the states of world before and after an execution of the service.

WSMO defines a Goal concept in addition to the Capability concept. SAWSDL also informally describes the Goal notion as a Web service request. In WSMO, a Goal is described by a postcondition. The functional description in the Goal expresses requirements and is used for searching and matching a Capability. If the post-condition in the Goal matches the one in the Capability, then the service is relevant. Such notion of Goal and Capability matching is part of the composability analysis.

Both OWL-S and WSMO also allow for a detailed functional description of a service by specifying a process specification. Such provision needs to be evaluated for complexity at the time of composability analysis. Semantic links between the service and ontological concepts of functions as in SAWSDL can be more computational friendly.

While OWL-S and WSMO define more expressive models for service descriptions, they lack provision for describing functions or domain-specific characteristics of the capability. For example, one order processing service may be able to process several order types including the new-item outbound order, inbound (return) order, and replacement order while another order processing service may only be able to process a new-item outbound order. Such differences in service scope must be known in order to effectively compose the services, yet current practices do not permit expression of the constraints on scope. In addition, a common semantic model for world states is needed when composing services from heterogeneous resources. Such problems have motivated several efforts to develop domain specific reference ontologies. Domain specific ontologies have been developed in the biological, biomedical, and financial domains. Ontologies for the industrial manufacturing domain are currently more fragmented [14-18], but recently, an Industrial 
Ontologies Foundry (IOF) [13] has been formed to develop coherent ontologies across manufacturing.

\subsection{Representing function}

Enhancing service description with better description of function is necessary for more precise composability analysis. This section of the paper reviews works in function representation theories and function modeling. Studies about function modeling and representation have been prevalent in the product design discipline to represent the "functional requirement".

First, we investigate the notion of function within the term "functional requirement." According to Glinz [19], there are two widely accepted definitions of the term, functional requirements, within the requirement engineering research area. The first definition emphasizes function. Suzanne and James [20] stated that a functional requirement specifies "requirement that specifies a function that a system or system component must be able to perform", while Sommerville [21] stated that functional requirement specifies "what the system should do". Despite using different terms, they commonly stated that functional requirement specifies function. The second definition emphasizes behavior. Anton [22] stated "functional requirements describe the behavioral aspects of a system". And Davis [23] stated "those requirements that specify the inputs (stimuli) to the system, the outputs (responses) from the system, and behavioral relationships between them; also called functional or operational requirements." According to IEEE 830 [24], functional requirements should define the fundamental actions required to process the inputs and generate the outputs. This literature provides the evidence that the notion of "function" is closely related with the term "behavior" and "action."

A number of existing works classify functions into two categories. Chittaro and Kumar [25] classified functions as the "operation function" or the "purposive function". Chkrabarti [26] classified them as the "intended behavior" or the "purpose". Charkrabarti later on with Bligh [27] classified them as the "action" or "effect". On the other hand, Deng [28] called them "action function" and "purpose function". However, the paper also raises an issue that the distinction between them is not clear-cut. This is because an action function may also contain design/purpose function, as it relates to intended and useful behavior. On the other hand, a purpose function may also require a certain action, even though this is not explicitly mentioned.

Chandrasekaran and Josephson [29] proposed a widely-accepted formal definition of function that cover all of the above notions. He classified functions as "device-centric" or "environment-centric". A device-centric function is formally represented with predicates over the variables associated with the internal structural elements of a system. (The term, system, here can be any of device, service, component and so on.) It corresponds to the behavior of a given system and covers the operational function in Chittaro and Kumar [25], the intended behavior in Chakrabarti [26], the action in Chakrabarti and Bligh [27], and the action function in Deng [28].

The environment-centric function, on the other hand, is represented with predicates (state) over elements (resource) external to the system and with a relationship to the device-centric function. Such relationship is called "Mode of Deployment". It allows for the assignment of a specific context to the device-centric function. For example, the environment-centric function of the electric lamp is "room illumination" when the lamp is placed (i.e., deployed) in a room with the switch turned on. Thus, the device-centric function of the electric lamp can be individuated as "illuminate something or somewhere". Chandrasekaran [30] explains that the device-centric 
function is the mean to achieving the environment-centric function and this statement also implies that the environment-centric function has a close relation with the "purpose". Thus, the environment-centric function covers the purposive function in Chittaro and Kumar [25], the purpose in Chakrabarti [26], the effect in Chakrabarti and Bligh [27], and the purpose function in Deng [28].

In section 3, we use Chandrasekaran and Josephson work to formally represent service and the characteristic of function the service possesses. Such representation is needed that goes beyond the mere input, output, and function label in today's popular service description standards.

\subsection{Approaches for automatic services composition}

In the past decade, a number of works in automatic services composition (specifically web services composition) appeared in literature. Artificial Intelligence (AI) techniques, specifically AI Planning techniques, were used for automatic services composition such as in [5-7, 35]. However, graph search methods were also popular such as in [36-38]. In the following sub-sections, an overview and characteristics of these two approaches are discussed.

\subsubsection{AI planning approaches}

Two kinds of AI planning-based approaches are distinguished. The first one is the domainindependent AI Planning approaches that try to solve general planning problem without reliance on domain-specific knowledge. The other is the domain-specific AI Planning approaches that use domain heuristics to help to solve the problem.

\subsubsection{Domain-independent planning}

In practice, it is not feasible to develop domain-independent planners that work in every possible domain. Thus, typically, most of the domain-independent planning approaches make simplifying assumptions to restrict the set of domains such as finite system, and deterministic outcome.

A class of the domain-independent planning approaches is the GraphPlan ${ }^{1}$ which is a generalpurpose planner for STRIPS ${ }^{2}$-style problems [31]. The operation of GraphPlan consists of two phases. In the first phase, a forward search is used to build a planning graph. In this phase, GraphPlan extends the planning graph forward from the initial state until a necessary (but insufficient) condition for plan exit is met. In the second phase, a regression search is performed to extract a sufficient plan. In this phase, backward search is performed from the goal, looking for a correct plan.

Another class of the domain-independent planning approach is Compilation-based Planning. These planners try to solve the planning problem by converting it into another classical planning problem such as SAT (Planning as Satisfiability) [32], CSP (Planning as Constraint Satisfaction Problem) [33], or ILP (Planning as Integer Linear Programming) [34]. Typical procedure of the compilation-based planning approaches is as follows: 1) set the maximum plan length to k, 2) encode the plan as a generic planning problems, 3) solve the problem using an off-the-shelf solver

\footnotetext{
${ }^{1}$ Despite its name GraphPlan is not considered a graph-based planning approach because the problem is not translated into a graph. Rather it dynamically generates possible solution graphs and prunes for a valid one similar to the Planning as Satisfiability.
}

${ }^{2}$ STRIPS stands for Stanford Research Institute Problem Solver. 
and 4) if a solution is found, decoded the generic planning problem into domain terms; otherwise, repeat the procedure after increasing the value of $\mathrm{k}$ until a plan is found.

\subsubsection{Domain-Specific Planning}

Domain-specific planning (DSP) is also known as configurable planning. DSP exploits one or a few planning recipes that are specific to a particular type or domain of problems. For example, a recipe for traveling to a distant destination may be 1) buying a ticket for a flight from the local airport to the remote airport, 2) taking a public transportation to the local airport, 3) flying to the remote airport, and 4) taking a public transportation to the final destination. Such recipes narrow down the search space rather than consider every combination of transportation modes, sequences, providers, and routes as required in the domain-independent method.

Hierarchical Task Network (HTN) Planning methods enable domain-specific planning. HTN planners divide the problem into tasks (activities), rather than goals and methods, and decompose tasks into subtasks. HTN Planners provide a construct to encode a recipe as a collection of methods and operators. Each recipe provides a way to solving a certain problem. As a result, the planning system does not necessarily have to repeatedly derive solutions. However, disadvantages of the HTN Planning is that writing a domain-specific knowledge can be more complicated than just writing classical operators.

Intuitively, AI planning approaches can be the solution for automatic services composition, which explains why a number of services composition researches are relying on them [35]. However, the computational complexity of the AI planning approaches is typically very high. Moreover, they do not guarantee that a solution will be found when one exists.

\subsubsection{Graph-based planning approaches}

In Graph-based planning approaches, composition problems are represented as a graph. Services, initial states, and goal states can be modeled as vertices, while input and output can be modeled as edges between vertices. This can also be done vice versa. Graph search algorithms find paths - a set of valid edges connecting the initial state to the goal state [36-38]. It is straightforward to construct an adjacent list or a matrix to represent a service network graph and obtain the shortest path from the source to the goal vertex using existing well-known shortest path finding algorithms such as the Bellman-Ford Algorithm and Dijkstra Algorithm. [39-42].

However, there are some limitations in the existing graph-based planning approaches. First, the existing approaches only support a graph with single input and single output per vertex. In addition, the existing methods only work with a single cost model and hard constraint associated with each of the edges. In this paper, realistic services composition problems with multiple cost models, hard constraints, as well as soft constraints (e.g., preferred vendors) are considered. To that effect, the existing methods for finding the shortest path cannot deal with these additional parameters.

\subsubsection{Automatic services composition}

While services composition can be viewed as a classical planning problem where AI planning approaches can be applied, graph-based planning approaches are more suitable from the perspective of computational efficiency. The main short-coming with AI planning is that they need to check for interleaving between sub-plans at every planning step. However, interleaving is less of an issue in the services composition problem because operators are more specific as the next service can be applied only if its pre-condition and input are compatible with the post-condition 
and output of the previous service. In other words, interleaving can be virtually prevented by constructing a composition-network graph from services compatibility and by applying cycle detection and elimination. As such, this paper extends and addresses the aforementioned shortcomings in graph-based planning. To that end, the next section first describes the formalism for service description necessary to represent the additional parameters by applying the function representation theories reviewed in Section 2.2. The representation is then used in the graph-based planning algorithm proposed in Section 4.

\section{Function and Service Representation Method}

In this section, we (1) consider what is needed to make services composable, (2) whether the condition differs in different types of services (software or hardware) and (3) whether the requirements for composition are functional or non-functional.

\subsection{Functional characteristics}

Functional characteristics of a service are related to the notion of function. For a clear definition of function, we investigated existing works in the function representation research area and found that there are a number of definitions in existing works as described in the related work section. These researches show that 'behavior' and 'effect' are two important aspects of the function notion.

The term 'behavior' means change objectively observable without any context. As we look at motorized toggle clamp as an example, it has a behavior that generates a clamping force when it is fed with the electrical energy. It also potentially generates other things such as a linear motion, heat, and noise, but let's focus on the clamping force. Figure 1 shows a behavioral modeling of the motorized toggle clamp. It has a Clamping function that takes electricity as an input and Clamping Force as an output.

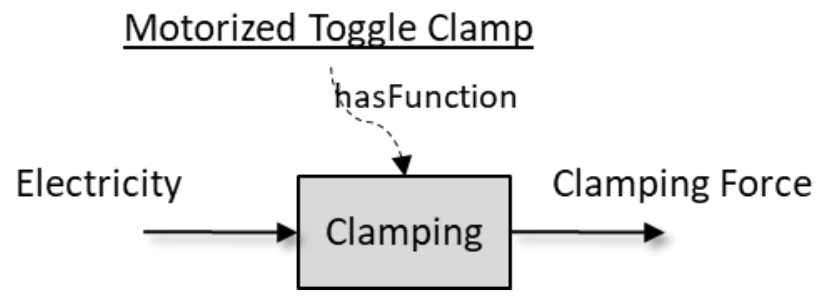

Figure 1. Composability with input and output

If we encapsulate the motorized toggle clamp as a Clamping service, then the service has the Clamping function with the respective input and output. Generally speaking, a service is a virtualization of a component or system and in some cases its location. In this example, the Clamping service abstraction hides from the service consumer/user what is actually providing the clamping force. Maybe it is a pneumatic toggle clamp instead of a motorized toggle clamp. In the case of a software service, it is common that the location is virtualized from the user, i.e., the user does not know which computer system and its location are used to execute the program.

In other words, the mechanism inside the service is not very much of a concern. What's important is the observable from the outside that are input, output, and some behavioral properties. Therefore, it is clear that the behavioral function of the service is described by the input to and output from the service; and they are essential functional characteristics of a service and services composability. That is, for services to be composed the output of one service must meet the input requirement of 
the other service to be composed. Thus, any service to be composed with the Clamping service must have the electricity as an output or the clamping force as an input. In some cases, the matching may not be exact. For instance, in the case that the output of one service is subsumed by the input of the next service, we can still say that the two services are composable. It should be noted that the representation of behavioral properties is addressed by the non-functional characteristics described in the next section.

Another important functional aspect of a service is the effect. The effect is a change the service has on its environment. Figure 2 shows an example.

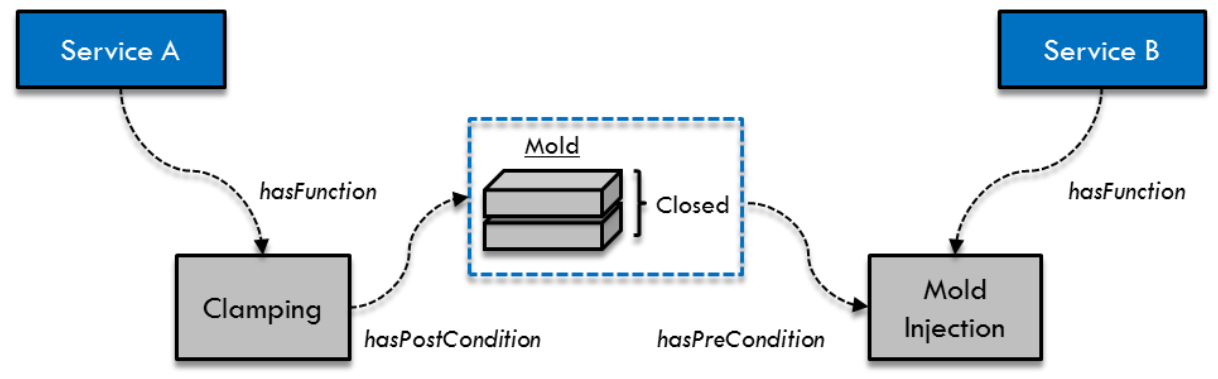

Figure 2. Composability with pre-condition and post-condition

Service A has a function called Clamping and Service B has a function called Mold Injection. The effect of Clamping is to push each of mold halves together and exerts sufficient force to keep them securely closed for the Mold Injection. The mold halves are resources entirely external to Service A, thus, according to Chandrasekaran and Josephson [29], the closing of the mold is an environment-centric function or effect of Service A. In other words, the effect is "Mold in the Closed state". Effect is an important functional characteristic for a services composition because it can satisfy a prerequisite of another service. As in the example above, prior to the material injection into the mold, the two halves of the mold must first be securely closed, hence the effect, "Mold in the Closed state" is a prerequisite to perform the Mold Injection function in Service B.

The effect can be manifested as a pre-condition or post-condition. If the effect must be present before performing a function within a service, the effect is represented as a pre-condition. If the effect occurs after an execution of a function within a service, then the effect is represented as a post-condition. Therefore, pre/post-conditions are conditions something not necessarily consumed or processed by the service.

\subsection{Non-functional characteristics}

Glinz (2007) [13] summarized several definitions of the non-functional requirement from the software engineering discipline. Commonly used terms are Property, Attribute, Quality, Constraint, and Performance. According to Glinz, there are not only terminological, but also major conceptual discrepancies in these definitions causing debates about how to express them and what should be considered functional or non-functional characteristics. To meet the objective of this research, we can generalize all functional and non-functional characteristics of a service as properties which can be manifested as qualities or constraints. A property can be expressed as a fixed value, a range, or an equation over some parameters.

For example, let's revisit the Injection Molding example that Service A provides a Clamping function and Service B provides a Mold Injection function. Both Service A and Service B may 
have the same non-functional property, mold pressure. Service A would express the property as a mold pressure quality, X. However, Service B would express the property as a constraint that the mold pressure must be greater than $\mathrm{Y}$ (which is derived from the injection pressure). Figure 3 illustrates this situation.

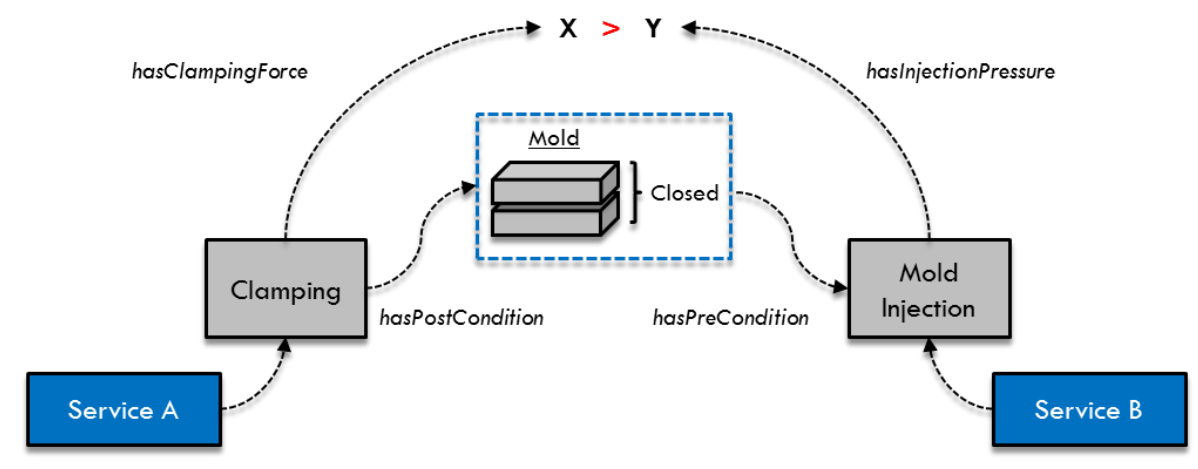

Figure 3. Composability with non-functional properties

\subsection{Service representation}

In 3.1 and 3.2, we identified necessary functional and non-functional characteristics that are relevant to the service composability. They include input, output, pre-condition, post-condition, and property. In this section, we provide a formal representation of the service.

To represent a service, we assume that there are a Resource and State ontology and a Service Property ontology. The Resource and State ontology defines the concepts and relationships used to represent the input, output, pre-condition, and post-condition. Specifically, the Resource is used to represent an artifact that is consumed (input) or produced (output) by the service. External artifacts that may effect or be effected by the operation of the service also can be represented using Resource. The pre/post-condition can be represented by the Resource's State. The Service Property ontology is used for representing the non-functional characteristics. Below, we provide the formal representation of the service used in the composability analysis algorithm described in the next section.

A service $S$ has six sets of parameters:

$S=\{F, I, O$, Pre, Post, Prop $\}$, where $^{3}$

$F=\{I, O$, Pre, Post, Prop $\}$ is the function of the service $S$, where

$I=\left\{I_{1}, I_{2}, I_{3}, \ldots\right\}$ is a set of inputs that are consumed by the service $S$.

- Input parameter $I_{i}=($ Resource, State $)$ is a pair of a Resource and its State where Resource and State are concepts defined in the Resource and State ontology respectively.

- Resource is mandatory but the State is optional. The State is specified only when there exists a constraint on the input, i.e., the input must be in a specific state.

\footnotetext{
${ }^{3}$ In some standards such as WSDL, a service can consist of multiple functions; but for the purpose of automatic services composition we can decompose the representation into a 1-1 relationship in term of observable function.
} 
- In order to invoke the service $S$, all input parameters must be provided ${ }^{4}$. $O=\left\{O_{1}, O_{2}, O_{3}, \ldots\right\}$ is a set of outputs that are produced by the function $F$.

- Output parameter $O_{j}=($ Resource, State) is a pair of a Resource and State where Resource and State are defined in the resource and state ontology respectively.

- The Resource is mandatory but the State is optional. The State is specified only when the output is in a specific state.

Pre $=\left\{\mathrm{Pre}_{1}, \mathrm{Pre}_{2}, \mathrm{Pre}_{3}, \ldots\right\}$ is a set of pre-conditions that are predicates that must always be satisfied in order that an invocation of the service $S$ yields the specified outputs and post-conditions. If any of the pre-conditions defined in the service $S$ is violated, the result of an invocation may not produce the specified outputs, post-conditions, and quality.

- Pre-condition parameter Pre P $_{k}=($ Resource, State $)$

- Both the Resource and State are mandatory.

- Note that the pre-condition is to describe the necessary condition on the external artifact not on the input consumed by the function.

Post $=\left\{\right.$ Post $_{1}$, Post $_{2}$, Post $\left._{3}, \ldots\right\}$ is a set of post-conditions that are the effects of an invocation of the service $S$.

- Post-condition parameter Post $=($ Resource, State $)$

- Both the Resource and State are mandatory.

- Note that the post-condition is to describe the effect on the external artifact not on the output.

Prop $=\left\{\right.$ Prop $_{1}$, Prop $_{2}$, Prop $\left._{3}, \ldots\right\}$ is a set of qualities or constraints (propositions) that are nonfunctional characteristics other than the input, output, pre-condition, and post-condition.

\section{Service Search and Composability Analysis Framework}

In this chapter, we describe our graph-based planning method for the service search and composability analysis. First the problem and the solution space are modeled as a network, which is then converted into an AND/OR graph. A search algorithm is then applied to the graph to find a set of services (a subgraph) that can traverse the graph from the initial condition to the goal condition, while minimizing the expected cost. Following such strategy, the problem modeling is first described in section 4.1 and the overall composability analysis process is described in 4.2.

\subsection{Problem modeling}

The problem modeling starts with the construction of a Composition Network (CN) from the available services (in a service registry). The $\mathrm{CN}$ represents a solution space. The initial and the goal conditions based on user's requirements are then incorporated into the $\mathrm{CN}$ by matching the conditions in the CN. A CN pruning is then applied. The result is transformed into an AND/OR graph that is suitable for the graph search method. The following subsections provide details of the problem modeling.

\footnotetext{
${ }^{4}$ This is a generalized representation. In practice, a service may have optional inputs. In such case, the representation of such service for the searching purpose shall be decomposed into several services each of which has all inputs required.
} 


\subsubsection{Composition network}

From the function and service representation in the previous section, the function and collectively the service are modeled as a vertex. By establishing the relationships between services based on their inputs, outputs, and pre/post-conditions form edges between the vertices, the result is an initial Composition Network (CN) graph. Figure 4 illustrates an example of an initial CN.

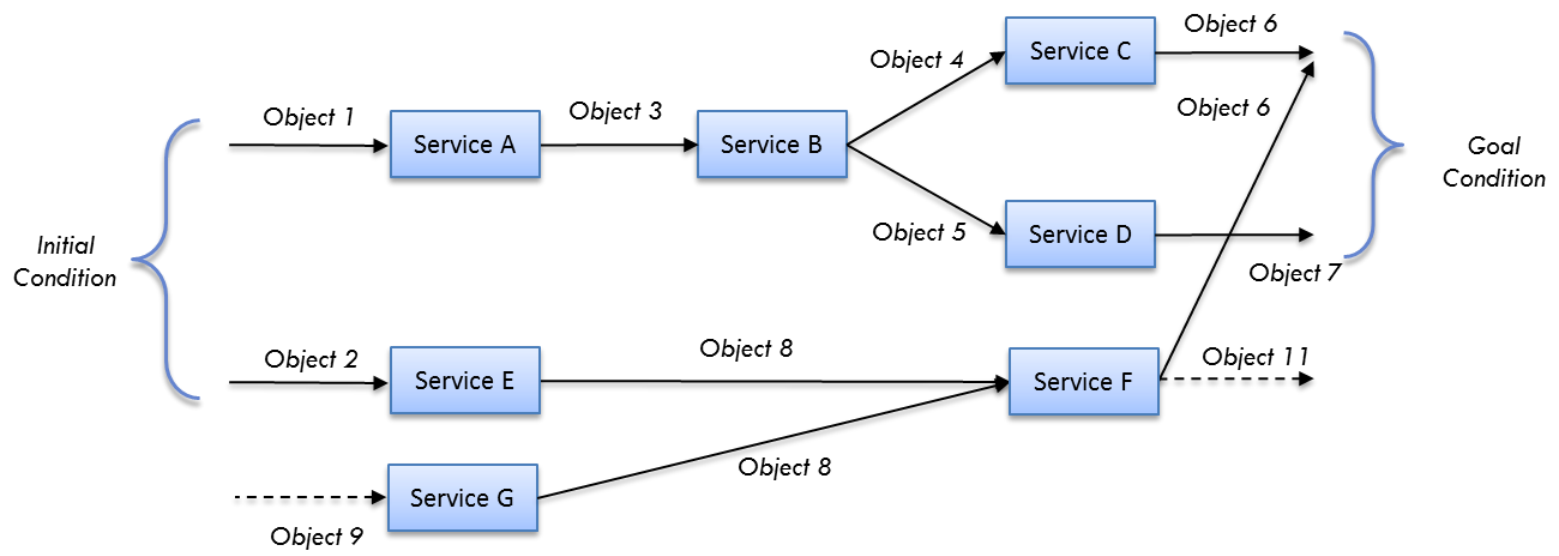

Figure 4. Example of Composition Network generated from services (Dotted arrows indicate objects unnecessary for the initial condition and goal)

In addition, the compatibility between the vertices can be quantified based on the constraints on properties as penalty configured by a user. The user can specify his/her own constraints on services to achieve the user's requirement. For instance, the user can specify a penalty for the total utilization costs of services for the given requirement. The search method will add the specified penalty to the total cost according to the services used in the result. For example, if the number of services (and also correspondingly functions) used is 4 and the user specifies 1 as a penalty for each service, the search method will add 4 to the total cost. The quantified compatibility penalty between the vertices is modeled as a cost function, $\operatorname{cost}\left(c_{r}\right)$, on the edge that connects the vertices. It should be noted that cost modeling can be a subject of further research. It can account for the difficulty of the service composition for example when there is a mismatch between message formats or security mechanisms. Thus, when quantifying the difficulty as a cost, the algorithm can consider the various characteristics of the services as well as user's preferences.

In the next step, the user requirement is incorporated into the $\mathrm{CN}$. The initial and the goal conditions based on user's requirements are matched within the initial CN. An initial vertex is added to the initial CN to link all services relevant to the initial conditions. Similarly, a goal vertex is added to the initial $\mathrm{CN}$ to link all services relevant to the goal conditions. The result is a $\mathrm{CN}$ shown as an example in Figure 5 - in this case Object 1 or 2 can be provided at the start while both Object 6 and 7 are required as the goal. Thus, the composability analysis problem is that of finding a set of vertices in the $\mathrm{CN}$ that are necessary to transit the initial vertices to the goal vertices while minimizing the sum of costs on the edges that connect these vertices. 


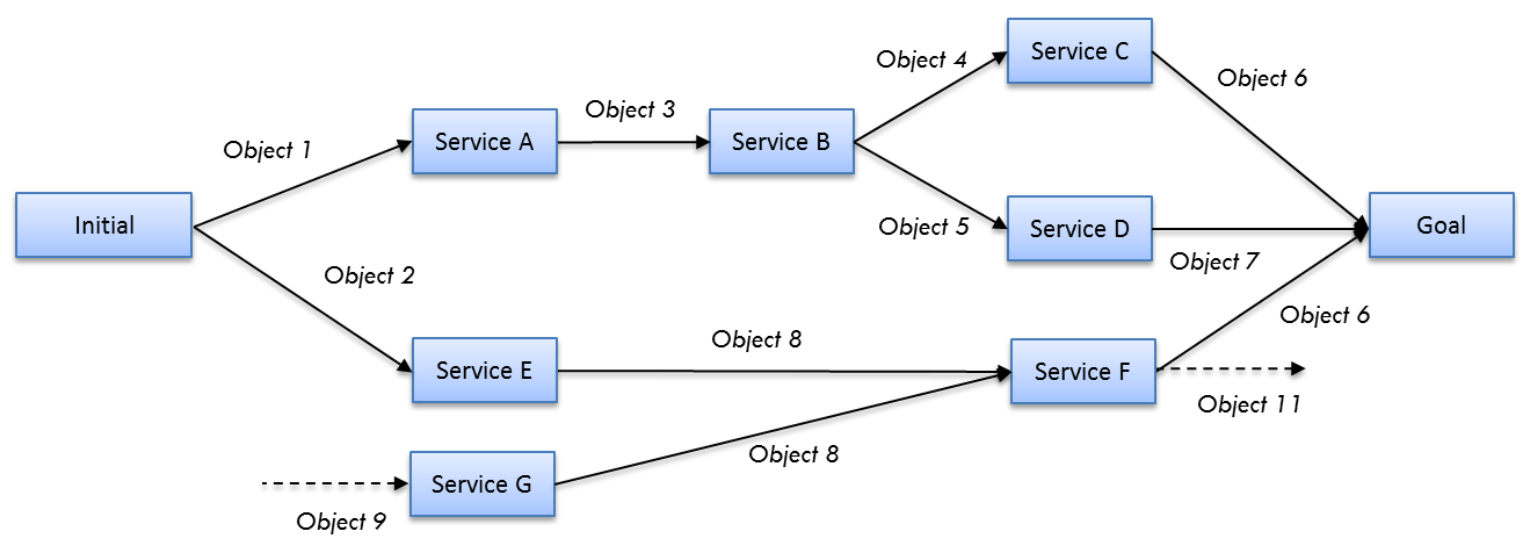

Figure 5. An example of composition network $(\mathrm{CN})$ with the initial and goal condition vertices added

The $\mathrm{CN}$ has embedded dependency and logical relationships. For example, Service $G$ has a dependency to object 9 in the example in Figure 5 because a vertex can be traversed (i.e., the service can be invoked) if and only if all the incoming edges are satisfied. In addition, there are AND/OR relationships between the incoming edges to the vertex. For example, in order to invoke Goal vertex, object 6 and object 7 must be provided. Thus, the two objects are logically ANDed. On the other hand, object 6 can be provided by either Service $C$ or Service F. That is, object 6 from Service $C$ and Service $F$ are logically ORed. These relationships need to be formally and explicitly represented in order to apply a graph-based search algorithm. The next section illustrates the conversion from the $\mathrm{CN}$ to an AND/OR graph.

\subsubsection{AND/OR graph conversion}

An AND/OR graph can be seen as a generalization of a directed graph. It contains a number of vertices and edges along with logical connectors that connect the vertices. Each connector in an AND/OR graph connects a set of vertices to a single vertex. A connector is said to be an AND connector, if there is a logical AND relationship. A connector is an OR connector, if there is a logical OR relationship.

Figure 6 below shows how the Service $C, D, F$, and Goal vertices in Figure 5 can be modeled as an AND/OR graph where two AND connectors connect edges from $\mathrm{C}$ and $\mathrm{D}$, and from $\mathrm{F}$ and $\mathrm{D}$, respectively, and the relationship between these two AND connectors is OR. Notice that Service $D$ shows up two times. In other words, there might be a node duplication issue when converting a $\mathrm{CN}$ to an AND/OR graph.

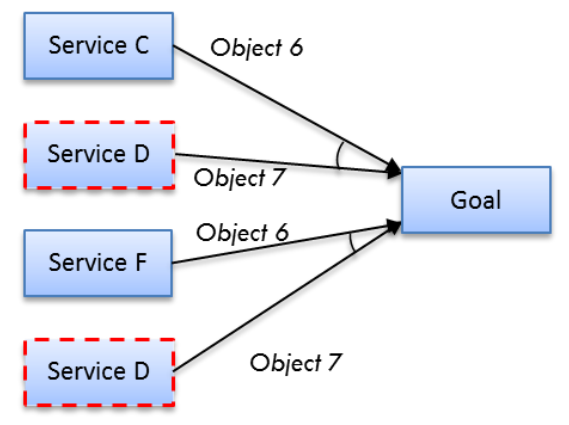

Figure 6. Example of AND/OR graph conversion with duplicative vertices 
In order to normalize the duplicative issue, we propose the following $\mathrm{CN}$ to AND/OR conversion method.

- Designate a service vertex as an AND vertex.

- Convert an object transmitted through an edge as an OR vertex.

- Convert the Goal vertex to a Starting AND vertex.

- Convert the Initial vertex to a Terminating OR vertex.

With the above conversion method, the $\mathrm{CN}$ in Figure 5 can be modeled as a normalized AND/OR graph as shown in Figure 13. The Goal vertex and Initial vertex are transformed into the Starting and Terminating vertex respectively. All the service vertices are represented as an AND vertex and the objects that are transmitted though edges are represented as an OR vertex.

The AND/OR graph representation encompasses all possible ways to achieve the user's requirement. Since each possible way corresponds to a solution subgraph in the AND/OR graph, the selection of the best way (minimal cost) can be viewed as a search problem.

\subsubsection{Problem formalization}

The followings are formal definitions of the composition network, AND/OR graph, and optimization problem.

Definition 1 (composition Network). A composition network, $C N=(V, E, w)$ is a weighted, directed graph, where $V$ is a set of vertices, $E$ is a set of edges, $\mathbb{R}$ is a set of real numbers, and $w$ is a set of weight (local cost) functions $w: E->\mathbb{R}$.

Each edge, $e \in E$, consists of four variables including source vertex $v_{s}$, target vertex $v_{t}$, and resource representing object $r$, and local $\operatorname{cost} c$ :

$e=\left\{v_{s}, v_{t}, r, c\right\}$.

The object $r$ represents a resource and its state that is transmitted through the edge.

Definition 2 (User's requirement). A user's requirement $R e q=\left\{R_{I}, R_{G}\right\}$ consists of a set of initial conditions $R_{I}$ and a set of goal conditions $R_{G}$.

RI consists of pairs of resource $r$ and its state $s$ :

$R_{I}=\left\{\left(r_{1}, s_{1}\right), \ldots\left(r_{k}, s_{k}\right) \mid r \in\right.$ Resource defined in the resource ontology and $s \in$ State defined in the state ontology $\}$.

$R_{G}$ also consists of pairs of resource $r$ and its state $s$ :

$R_{G}=\left\{\left(r_{1}, s_{1}\right), \ldots\left(r_{j}, s_{j}\right) \mid r \in\right.$ Resource defined in the resource ontology and $s \in$ State defined in the state ontology $\}$

$R_{I}$ and $R_{G}$ are represented as the Initial and Goal vertices respectively in the composition network. Therefore, $v_{I}$ has $R_{I}$ as outgoing edges and does not has any incoming edge, while $v_{G}$ has $R_{G}$ as an incoming edge and does not have any outgoing edge.

Definition 3 (AND/OR graph). An AND/OR graph, $A O=\left(V_{a n d}, V_{o r}, E^{\prime}, w\right)$ is a weighted, directed graph, where, $V_{\text {and }}$ is a set of AND vertices, $V_{\text {or }}$ is a set of OR vertices, $E^{\prime}$ is a set of edges, $\mathbb{R}$ is a set of real numbers, and $w$ is a set of weight (local cost) functions $w: E^{\prime}->\mathbb{R}$ as in Definition 1. 
Each AND vertex, $v_{\text {and }} \in V_{\text {and }}$, has one or more edges directed to OR vertices. The OR vertices are called the immediate successors of $v_{\text {and }}$ and the edges have logical AND relationship such that all the OR vertices must be provided to achieve the $v_{\text {and }}$.

Each OR vertex, $v_{o r} \in V_{o r}$, has one or more edges directed to AND vertices. The AND vertices are called the immediate successors of $v_{o r}$ and the edges have logical OR relationship such that any one of the AND vertices enables the OR vertex.

Each edge $e^{\prime} \in E^{\prime}$ consists of two variables including source vertex $v_{s}$, and target vertex $v_{t}$ :

$e^{\prime}=\left\{v_{s}, v_{t}\right\}$

Definition 4 (Composition Network as an AND/OR graph). A Composition Network is converted into an AND/OR graph as follows:

$v \in V$ in the composition network is converted into $v_{\text {and }} \in V_{\text {and }}$ in AND/OR graph.

$v_{I} \in V$ in the composition network is converted into the terminal vertex in AND/OR graph.

$v_{G} \in V$ in the composition network is converted into the starting vertex in AND/OR graph.

e.o $(e \in E)$ in the composition network is converted into $v_{o r} \in V_{o r}$ in AND/OR graph. Note that there must be a single OR vertex for each object, even though there might exist multiple edges that have same object.

$e \in E$ in the composition network is converted into two edges $e_{1} \in E^{\prime}$ and $e_{2} \in E^{\prime}$ ' in AND/OR graph:

$$
\begin{aligned}
& e_{1}=\left(e \cdot v_{t}, v_{o r}\right) . \\
& e_{2}=\left(v_{o r}, e . v_{s}\right) .
\end{aligned}
$$

Definition 5 (Solution Graph). Given an AND/OR graph $A O$, let $s$ be the starting vertex and $t$ be the terminal vertex. A solution graph $s g$ is a finite sub-graph of $A O$ that satisfies the followings: $s$ is a root of $s g$; for $\forall v \in V_{\text {and }}$, all of $v$ 's immediate successors are in $s g$; for $\forall v \in V_{o r}$, only one of $v$ 's immediate successors is in $s g$; and every directed path starts from $s$ ends with $t$.

Definition 6 (Minimum Cost Solution Graph). Given an AND/OR graph $A O$, let $s$ be a start vertex and $t$ be a terminal vertex. The minimum cost solution graph is a solution graph with the minimum of the sum of the weights (local costs) on the constituent edges.

\subsection{Composability analysis}

The search method consists of three main steps 1) the composition network pruning and cost estimation; 2) AND/OR graph transformation; and 3) search for the minimum cost subgraph. The following subsections describe each of these steps.

\subsubsection{Composition network pruning and cost estimation}

After generating $\mathrm{CN}$, the vertices in the graph are topologically sorted. The topological sorting algorithm eliminates cyclical dependencies in the graph and orders the vertices according to their precedence relationship in a linked-list. It is described in section 4.2.1.2. The resulting linear ordering of vertices has no duplicates. In the next step, all vertices are initialized. Vertex initialization is an initial cost assignment described in 4.2.1.3. Then, a cost estimation algorithm 
call Relaxation is applied. It assigns to each vertex the estimated cost for transitioning into the vertex. After the first relaxation, a pruned composition network is produced where unnecessary vertices and edges will be screened out. However, there is a possibility for an overestimation. We can address the overestimation by applying another cost-adjustment relaxation algorithm on the pruned composition network. Detail of the cost adjustment algorithm is given in section 4.2.1.5.

The proposed algorithms are based on the DAG-SHORTEST-PATHS algorithm [47] with specific methods and data structures extended for the composition network analysis. The extended methods are represented with asterisk in the pseudo code below.

\subsubsection{Notations}

We use the following notations as well as the definitions in Section 4.1.3 to describe all the pseudo codes of the procedures.

- Vertex $v$ has a set of inputs $I$, outputs $O$, incoming edges $E_{i}$, outgoing edges $E_{o}$, and cost $c$ from the initial vertex:

$$
v=\left(I, O, E_{i}, E_{o}, c\right)
$$

- Note that the inputs and outputs are explicitly defined in each vertex, while the incoming and outgoing edges are implicitly defined by the dependency between a source vertex and its target vertex.

- $\quad I$ and $O$ consists of pairs of resource $r$ and cost $c_{r}$ of obtaining the resource from the initial vertex.

$$
\left\{\left(r_{1}, c_{r 1}\right), \ldots\left(r_{k}, c_{r k}\right) \mid r_{k} \in \text { resources defined in the resource ontology }\right\}
$$

- $\quad E_{i}$ consists of source vertex $v_{s}$, resource $r$, and cost of obtaining the resource from the initial vertex $c_{r}$.

$$
E_{i}=\left\{\left(v_{s l}, r_{1}, c_{r l}\right), \ldots\left(v_{s j}, r_{k}, c_{r k}\right) \mid r_{k} \in \text { resources defined in the resource ontology }\right\}
$$

- The cost from the initial vertex of vertex $v, v \cdot c$ is the sum of the cost of each unique input resource in the set of incoming edges of $v, v \cdot E_{i}$ :

$$
\text { v.c }=\sum_{i=1}^{k} c_{r i}
$$

- Some of the incoming edges may have the same resource with different source vertex. Thus, the cost $c_{r}$ of the input is the minimum cost of the incoming edges that have $r$ as a resource: $c_{r}=\operatorname{Min}$ (the costs of the incoming edges that has $r$ as a resource)

- $\quad E_{o}$ consists of target vertex $v_{t}$, resource $r$, and cost of obtaining the resource from the initial vertex $c_{r}$.

$$
E_{o}=\left\{\left(v_{t 1}, r_{1}, c_{r 1}\right), \ldots\left(v_{t m}, r_{k}, c_{r k}\right) \mid r_{k} \in \text { resources defined in the service ontology }\right\}
$$

- Each edge $e \in E$ consists of four variables including source vertex $v_{s}$, target vertex $v_{t}$, resource $r$, and local cost $c$ of the resource. The resource $r$ represents an object that is transmitted through the edge:

$$
e=\left(v_{s}, v_{t}, r, c\right)
$$


The pseudo code below shows the overall procedure of the composition network pruning and cost estimation method using the notation.

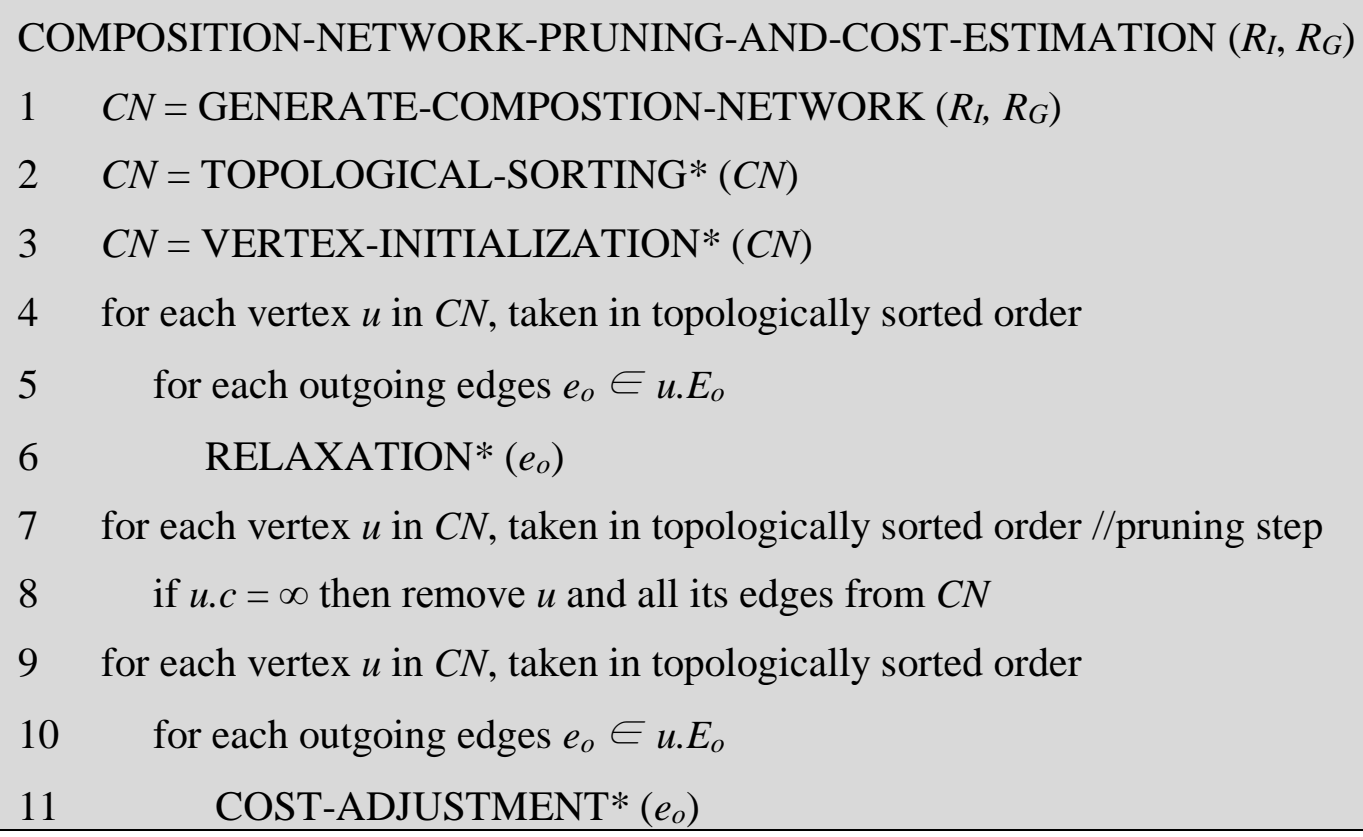

\subsubsection{Topological sorting}

The topological sorting orders all the vertices linearly. Suppose that the composition network has an edge $\left(v_{1}, v_{2}\right)$. Then, $v_{1}$ appears before $v_{2}$ in the order after the topological sorting. One issue in the topological sorting is that if the graph contains a cycle, then linear ordering of the vertices is not possible. Therefore, we have to check whether the composition network contains a cycle. The cycle detection can be done by the depth first search. A directed graph is acyclic if and only if a depth-first search of the graph yields no back edges. If the graph contains a cycle, then we have to make the composition network acyclic through the CREATE-STRONGLY-CONNECTEDVERTEX method as described in [44]. Figure 7 below shows the result of the topological sorting on the $\mathrm{CN}$ presented in the Figure 5.

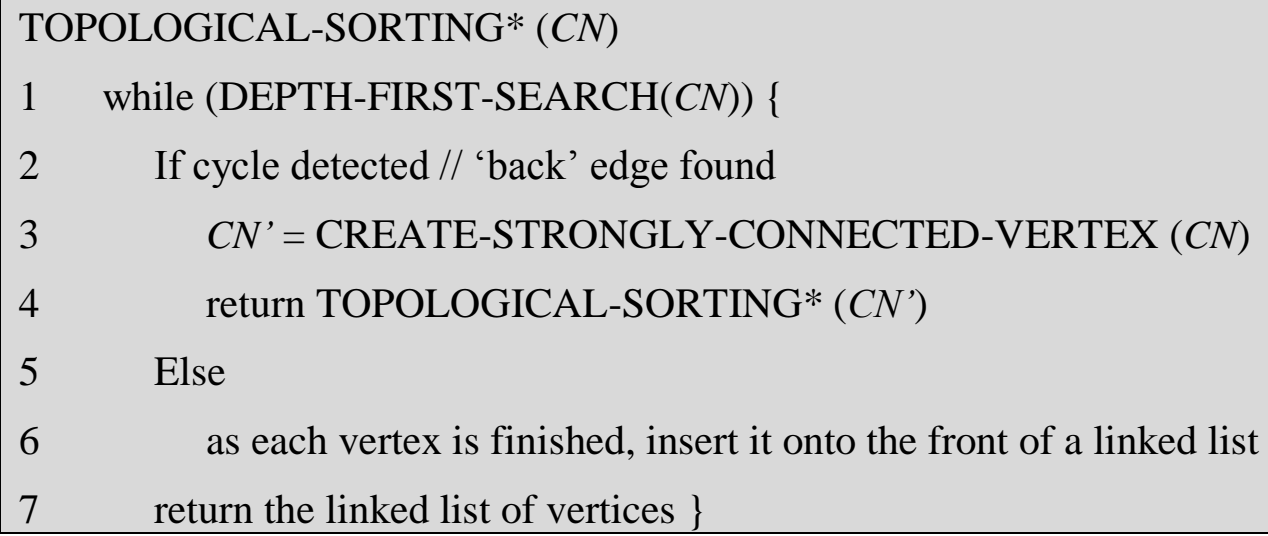




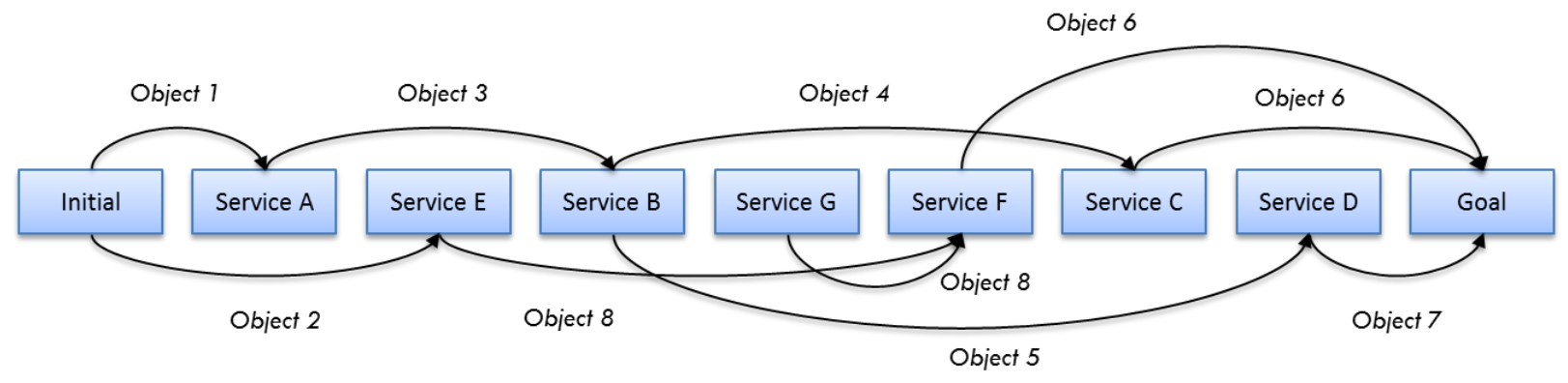

Figure 7. Topologically sorted CN.

\subsubsection{Vertex initialization}

In this step, the costs in the input set, and in the incoming/outgoing edges of the vertex are initialized following the pseudo code below. The initial vertex will have 0 as a cost, while others are initialized with $\infty$. Figure 8 shows the result of the vertex initialization. The white boxes in the bottom represent the cost of each corresponding vertex above.
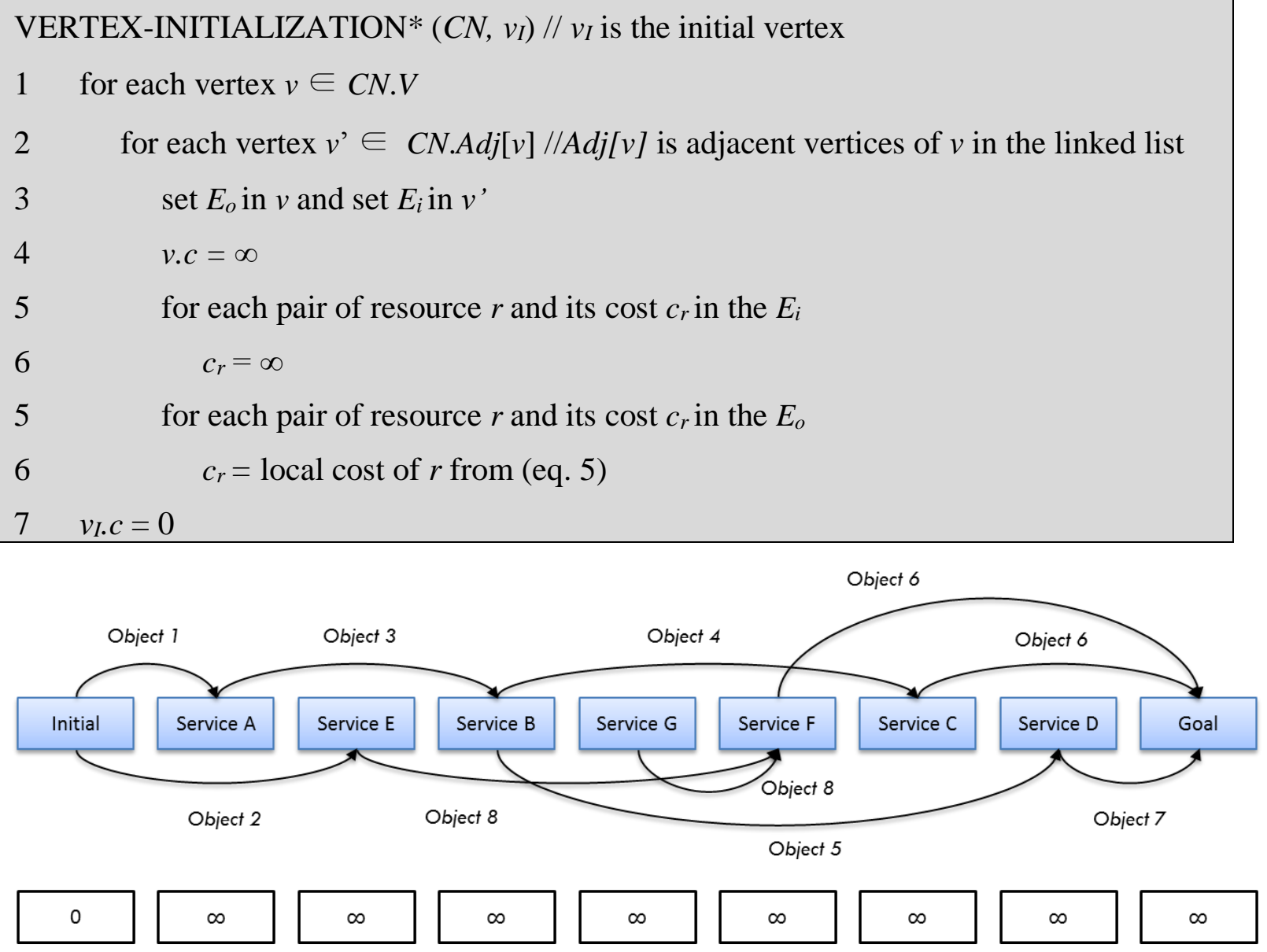

Figure 8. CN with the Vertex Initialization 


\subsubsection{Relaxation}

The algorithm for composition network pruning and cost estimation uses the relaxation technique. Each vertex maintains an upper bound of the cost from source vertex. The upper bound of the cost is represented as $c_{t}$. The $c_{t}$ of each vertex has been initialized as $\infty$ in the vertex initialization step. The relaxation on an edge $(u, v)$ checks whether the cost to $v$ can be improved by going through $u$. If the cost can be improved, then $c_{t}$ of $v$ is updated. The details of the checking and updating procedures are described in the following pseudo code.

\section{RELAXATION* $\left(e_{o}\right)$}

1 Look up $e \in E$ using $e_{o} . v_{t}$ and $e_{o} . r$

$2 v_{s}=e \cdot v_{s}, v_{t}=e \cdot v_{t}$

$3 \quad c_{\text {new }}=v_{s} \cdot c+e_{0} \cdot c$

4 Get $\operatorname{cost} c_{r}$ of the resource in $v_{t} . E_{i}$ using $e_{o} . r$ as a key

5 if $c_{\text {new }}<c_{r}$ then update $c_{r}=c_{\text {new }}$ in $v_{t .} E_{i}$ and update $v_{t .} c$ in eq. 1 using new $c_{r}$ via eq. 4.

Figure 9 below illustrates the result of the relaxation assuming the local cost of every resource is 1. The number in the white boxes represents the cost from the initial vertex to each vertex. There is one vertex that has $\infty$ as a cost. Thus, the vertex as well as the edges of the vertex are eliminated in the pruning step. Figure 10 shows the composition network left before the cost adjustment is applied.

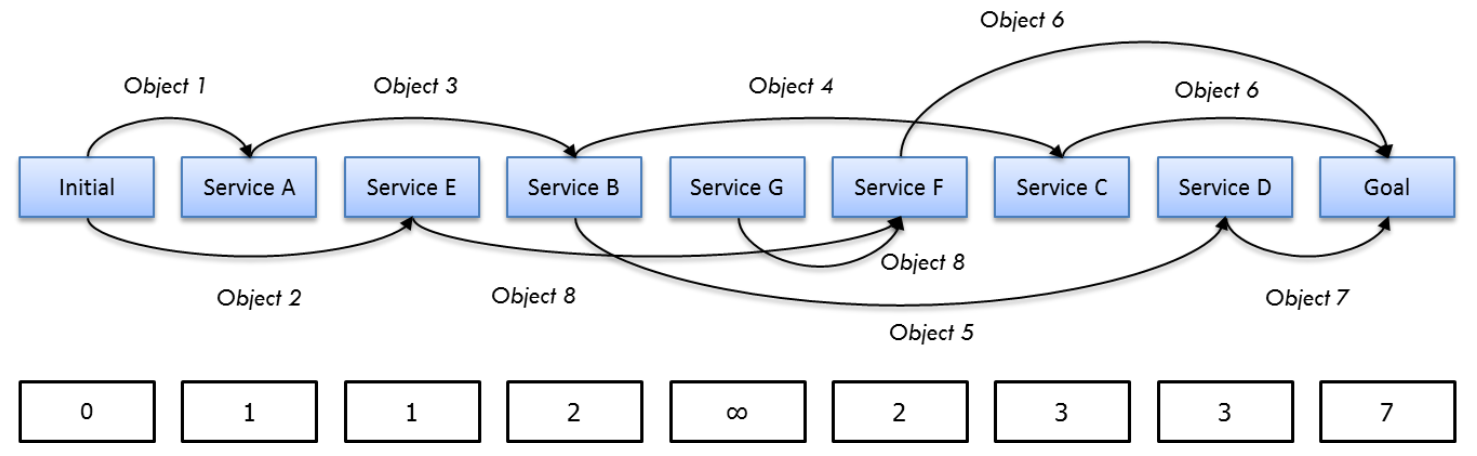

Figure 9. Result of the relaxation 


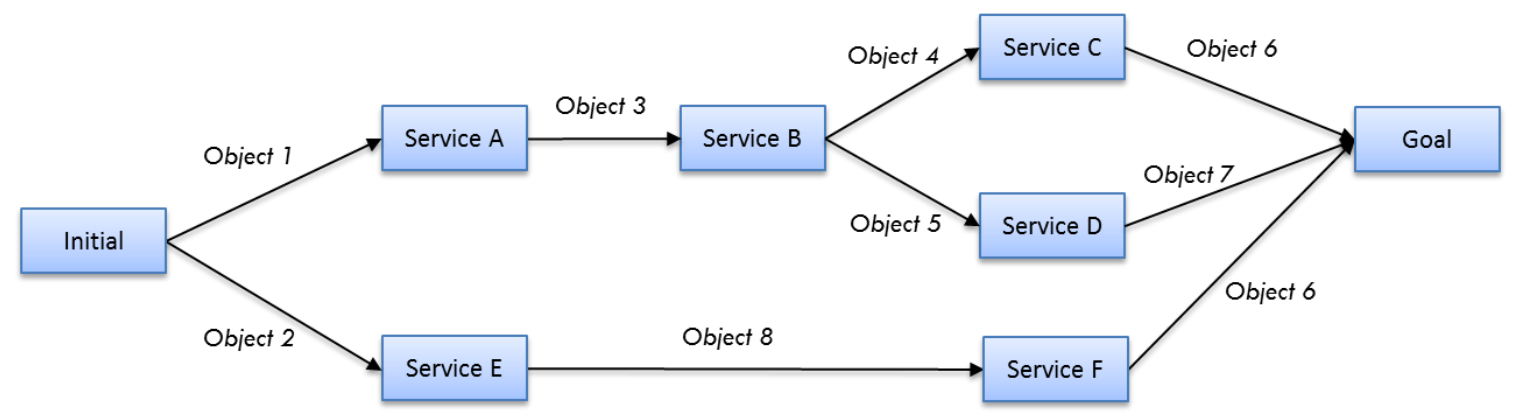

Figure 10. Result of composition network pruning

\subsubsection{Cost-adjustment}

The resulting cost of each vertex in the previous section may be overestimated, specifically when there are overlaps between paths. For example, let's take a look at the illustration shown in Figure 11. Once again assuming that the local costs of all edges are 1 . There are three paths from the start vertex to the end vertex. For Object 7, there exists only one path while for Object 6 , there are two different paths. The minimum cost path for Object 6 is Path 3 that costs 3 . Since there is only one path for Object 7, if we just aggregate the minimum cost paths, then Path 2 and Path 3 will be chosen, and the total cost would be 7 . However, we can reduce the total cost to 6 by choosing Path 1 instead of Path 3 even though the cost of the Path 1 is greater than the Path 3, because Path 1 and Path 2 overlap. Thus, the cost of getting to the End vertex by the relaxation procedure was overestimated. In order to avoid the overestimation in the example, the cost up to Service B should be shared by Service $C$ and $D$. That is, the cost of the shared path should not be added in both of the branching paths.

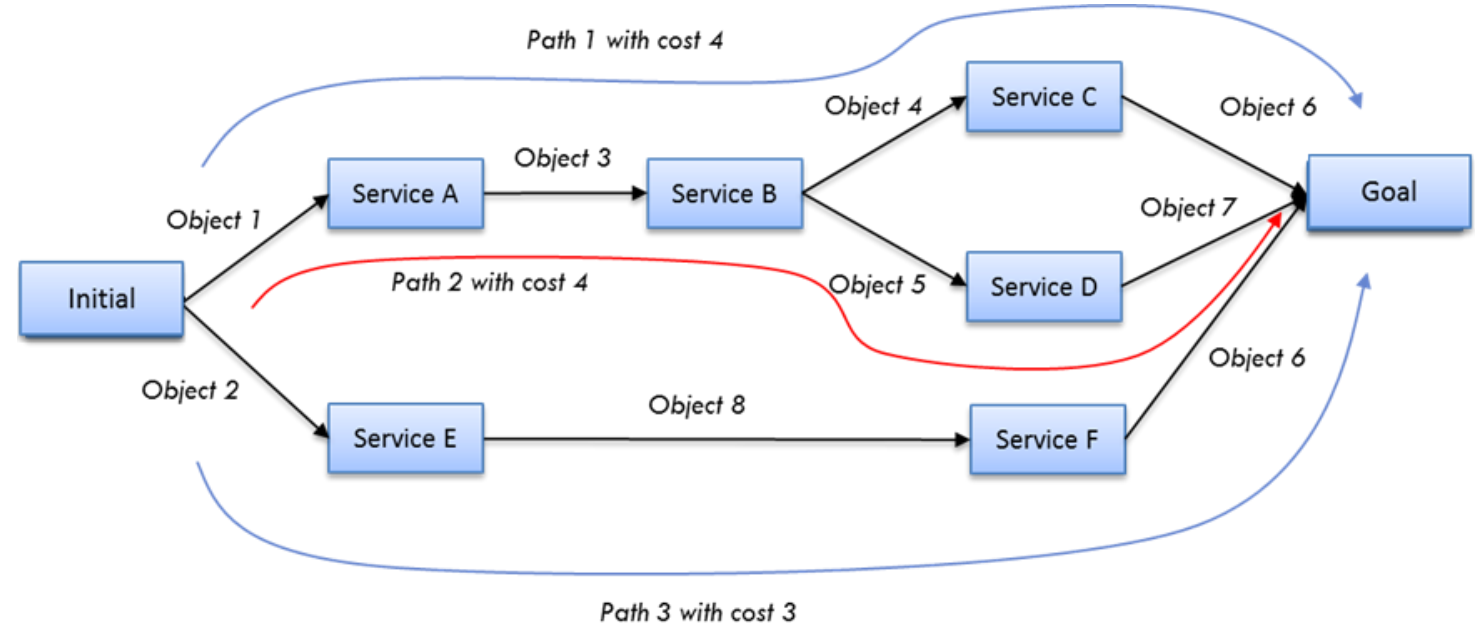

Figure 11. Cost over estimation when branches exist.

To address this issue, the following cost-adjustment method is proposed. The cost-adjustment divides the cost up to the precedent vertex by the degree of the outgoing edges when relaxing the adjacent vertices of the precedent vertex. 


\section{COST-ADJUSTMENT* $\left(e_{o}\right)$}

1 Look up $e \in E$ using $e_{o .} v_{t}$ and $e_{o . r}$

$2 v_{s}=e \cdot v_{s}, v_{t}=e \cdot v_{t}$

$3 \quad c_{\text {new }}=\frac{v_{s . c}}{\left.\text { (outgoing degree of } v_{s}\right)}+e_{0} \cdot c$

4 Get cost $c_{r}$ of the resource in $v_{t} . E_{i}$ using $e_{o} . r$ as a key

5 if $c_{\text {new }}<c_{r}$

6 then update $c_{r}=c_{\text {new }}$ in $v_{t} . E_{i}$ and update $v_{t .} . c$ in eq. 1 using new $c_{r}$ via eq. 4.

For example, when processing the Service $B$ in the Figure 11 the Service $C$ will be relaxed by the following way:

$($ cost up to Service $B) /($ the outgoing degree of Service $B)+($ edge cost $)=2 / 2+1=2$.

Figure 12 below shows the result of the cost-adjustment.

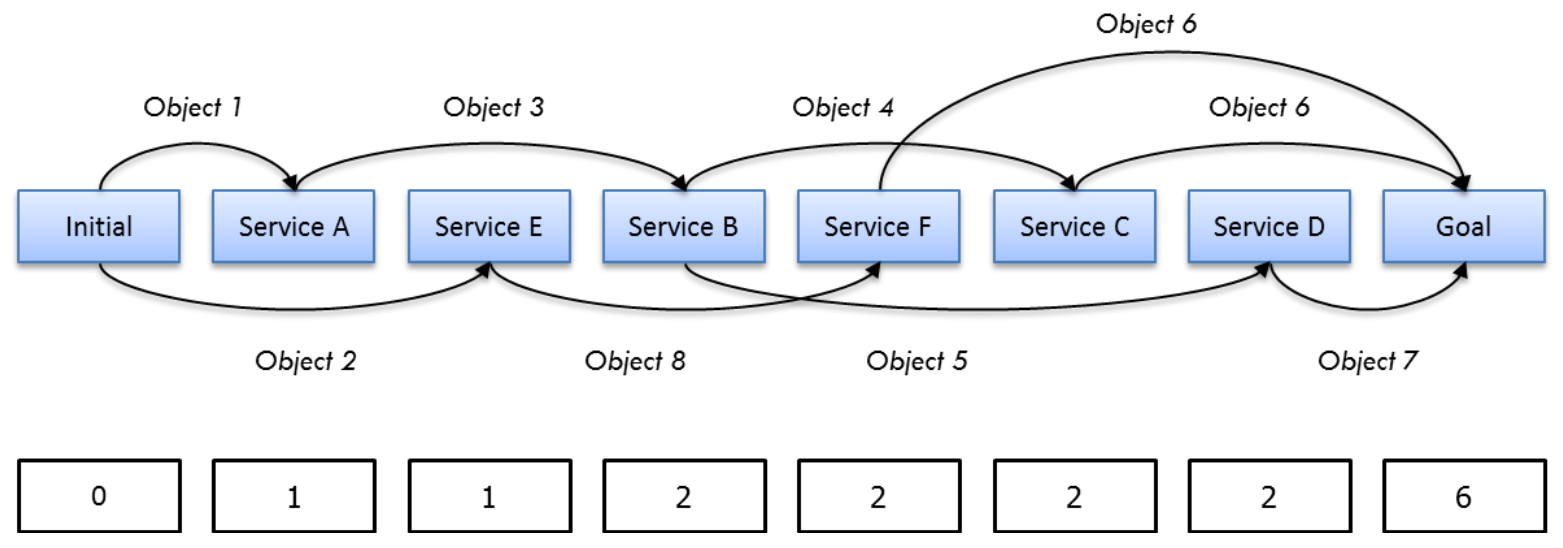

Figure 12. Result of Cost-adjustment

The following definition, theorem and corollary present how the cost-adjustment can guarantee that the estimated cost is always lower than or equal to the actual minimum cost.

Definition 7 (Branching Vertex). Let $G=(V, E, w)$ be a weighted, directed graph with weight (local cost of edges) function $w: E \rightarrow \mathbb{R}$. Any $v \in V$ that satisfies the followings is defined as a branching vertex:

The size of $v \cdot E_{o}$ is greater than 1 such that $v$ has at least two outgoing edges regardless of the object on the edges.

$v$ has precedent vertices that provide all inputs that are required to invoke $v$.

$v$ is not the start vertex.

Theorem 1 (Upper-bound without any branching vertices) 
Let $G=(V, E, w)$ be a Composition Network. Assume that the graph is relaxed by RELAXATION*. Let $G^{\prime}=\left(V^{\prime}, E^{\prime}, w\right)$ be the minimum cost solution graph of $G$ that has $s \in V^{\prime}$ as the start vertex and $v \in V^{\prime}$ as the terminal vertex. Assume that $G^{\prime}$ does not have any branching vertex, then $v \cdot c_{t}=\delta(s, v)$ after the COST-ADJUSTMENT*, where $v \cdot c_{t}$ is the estimated cost of $G^{\prime}$ and $\delta(s, v)$ is the actual minimum cost of $G^{\prime}$.

\section{Proof}

Assume that $v$ has $n$ inputs, where $n \geq 1$. Then, $\delta(s, v)=c\left(s g_{1}\right)+c\left(s g_{2}\right)+\ldots+c\left(s g_{n}\right)$, where $c\left(s g_{k}\right)$ is the cost of the minimum cost solution graph for the input $k$ of $v$.

There is no overlapping vertices or edges between the solution graphs because $G$ ' does not have any branching vertex. Thus, taking the minimum cost solution graph for each input of $v$ always guarantees the total minimum cost of $v$ as the RELAXATION method does. Since there is no branching vertex, COST-ADJUSTMENT* is exactly the same with the RELAXATION. Thus, $v . c_{t}$ $=\delta(s, v)$.

Theorem 2 (Upper-bound with one branching vertex)

Let $G=(V, E, w)$ be a Composition Network. Assume that the graph is relaxed by RELAXATION*. Let $G^{\prime}=\left(V^{\prime}, E^{\prime}, w\right)$ be the minimum cost solution graph of $G$ that has $s \in V^{\prime}$ as the start vertex and $v \in V^{\prime}$ as the terminal vertex. Assume that $G^{\prime}$ has only one branching vertex $u$ $\in V^{\prime}$ and $u$ has $n$ outgoing edges, where $n>1$. Then, $v . c_{t}<\delta(s, v)$ after the COSTADJUSTMENT*, where $v \cdot c_{t}$ is the estimated cost of $G^{\prime}$ and $\delta(s, v)$ is the actual minimum cost of $G^{\prime}$.

\section{Proof}

Since there is no branching vertices in the precedent vertices of $u, \delta(s, u)=u . c_{t}$ by Theorem 1 .

Let $G$ ' $\left(V^{\prime \prime}, E^{\prime \prime}, w\right)$ be the minimum cost sub-solution graph of $G$ ' that has $s$ as the start vertex and $u$ as the terminal vertex. Then, $E$ ' ' $\subset E$ ' and we can represent $\delta(s, v)$ by the following.

$\delta(s, v)=\left(\right.$ the sum of the weights on $\left.\forall e^{\prime} \in E^{\prime \prime}\right)+X$, where $X$ is the sum of the weights on $\forall e \in E^{\prime}-E^{\prime \prime}=\delta(s, u)+X$

After COST-ADJUSTMENT*, add $\frac{u \cdot c_{t}}{n}$ to the weights of each outgoing edges of $u$ and set 0 for $\forall$ $e^{\prime} \in E^{\prime}$. Let $E_{o}$ be the set of the outgoing edges of $u$ with $n$ members.

If $\left(\forall e \in E_{o}\right) \in E^{\prime}$, then

$$
v \cdot c_{t}=\left(\frac{u \cdot c_{t}}{n}\right) * n+X=u \cdot c_{t}+X=\delta(s, u)+X=\delta(s, v)
$$

Else if $\left(\exists e \in E_{o}\right) \in E^{\prime}$, then 


$$
\begin{aligned}
v \cdot c_{t} & =\left(\frac{u \cdot c_{t}}{n}\right) * k+X, \text { where } k<n \\
& <u . c_{t}+X=\delta(s, u)+X=\delta(s, v)
\end{aligned}
$$

Thus, $v . c_{t} \leq \delta(s, v)$

Theorem 3 (Upper-bound with multiple branching vertices)

Let $G=(V, E, w)$ be a Composition Network. Assume that the graph is relaxed by RELAXATION*. Let $G^{\prime}=\left(V^{\prime}, E^{\prime}, w\right)$ be the minimum cost solution graph of $G$ that has $s \in V^{\prime}$ as the start vertex and $v \in V^{\prime}$ as a terminal vertex. Then, $v . c_{t} \leq \delta(s, v)$ after the COSTADJUSTMENT*, where $v . c_{t}$ is the estimated cost of $G^{\prime}$ and $\delta(s, v)$ is the actual minimum cost of $G^{\prime}$, and this invariant is maintained over any number of branching vertices in $G^{\prime}$.

\section{Proof}

We prove the invariant $v . c_{t} \leq \delta(s, v)$ by induction over the number of branching vertices in $G^{\prime}$. Let the number of branching vertices in $G^{\prime}$ be $k$. For the basis $(k=1), v . c_{t} \leq \delta(s, v)$ is certainly true by Theorem 2.

For the inductive step, consider there are $n$ branching vertices in $G^{\prime}$. By the inductive hypothesis, $v . c_{t} \leq \delta(s, v)$ for all the number of branching vertices equal or less than $n$ in $G^{\prime}$.

Let $G_{n+1}=\left(V_{n+1}, E_{n+1}, w\right)$ be the minimum cost sub-solution graph of $G$ ' that has $s$ as the start vertex and $u_{n+1}$ as the terminal vertex, where $u_{n+1}$ is the last branching vertex in topological order in $G^{\prime}$. Let $U=\left\{u_{1}, \ldots, u_{n}\right\}$ be other branching vertices in $G^{\prime}$.

If ( $\forall u \in U) \notin V_{n+1}$, then $u_{n+1}$ does not have any precedent branching vertices. Thus, $v . c_{t} \leq \delta(s$, v) by Theorem 2 .

If ( $\exists u \in U) \in V_{n+1}$, let $u_{m}$ be the last branching vertex in $G_{n+1}$ where $1 \leq m \leq n$. Since $G_{n+1}$ has the number of branching vertices equal or less than $n$, by the inductive hypothesis, $u_{n+1} . c_{t} \leq$ $\delta\left(s, u_{n+1}\right)$.

Since $E_{n+1} \subset E^{\prime}$, we can represent $\delta(s, v)$ by the following.

$\delta(s, v)=$ (the sum of the weights on $\left.\forall e^{\prime} \in E_{n+1}\right)+X$, where $X$ is the sum of the weights on $\forall e \in E^{\prime}-E_{n+1}$.

$$
\begin{aligned}
& =\delta\left(s, u_{n+1}\right)+X \\
& \geq u_{n+1} . c_{t}+X
\end{aligned}
$$

After COST-ADJUSTMENT* on $u_{n+1}$, add $\frac{u_{n+1} \cdot c_{t}}{j}$ to the weights of each outgoing edges of $u_{n+1}$, where $j$ is the outgoing degree of $u_{n+1}$ and set 0 for $\forall e \in E_{n+1}$. Let $E_{o}$ be the set of the outgoing edges of $u_{n+1}$.

$$
\text { If }\left(\forall e \in E_{o}\right) \in E^{\prime} \text {, then }
$$




$$
v \cdot c_{t}=\left(\frac{u_{n+1} \cdot c_{t}}{j}\right) * j+X=u_{n+1} \cdot c t+X \leq \delta(s, v)
$$

Else if $\left(\exists e \in E_{0}\right) \in E^{\prime}$, then

$$
\begin{aligned}
v \cdot c_{t} & =\left(\frac{u_{n+1} \cdot c_{t}}{j}\right) * k+X, \text { where } k<j \\
& <u_{n+1} \cdot c_{t}+X \leq \delta(s, v)
\end{aligned}
$$

Thus, the invariant is maintained.

The following corollary proves that the estimated cost resulting from the cost-adjustment process is an admissible heuristic for searching AND/OR graph that is converted from a given $\mathrm{CN}$. Thus, the estimated cost can be used as a heuristic function for the search algorithm in section 4.2.2.

\section{Corollary 1}

The estimated cost resulting from the cost-adjustment process is an admissible heuristic for AND/OR graph search.

\section{Proof}

An admissible heuristic is used to estimate the cost of reaching the goal state in an informed search algorithm. In order for a heuristic to be admissible to the search problem, the estimated cost must always be lower than or equal to the actual cost of reaching the goal state [43]. By the Theorem 1, 2,3 , the estimated cost is always lower than or equal to the actual cost. Thus, the estimated cost resulting from the cost-adjustment process is an admissible heuristic.

\subsubsection{Transformation to AND/OR graph}

After the composition network pruning and cost estimation, the AND/OR graph conversion method defined in section 4.1.2 is applied to the composition network. Figure 13 below shows the transformed AND/OR graph from CN in Figure 12. Each AND vertex has an estimated cost that is represented in the parenthesis. 


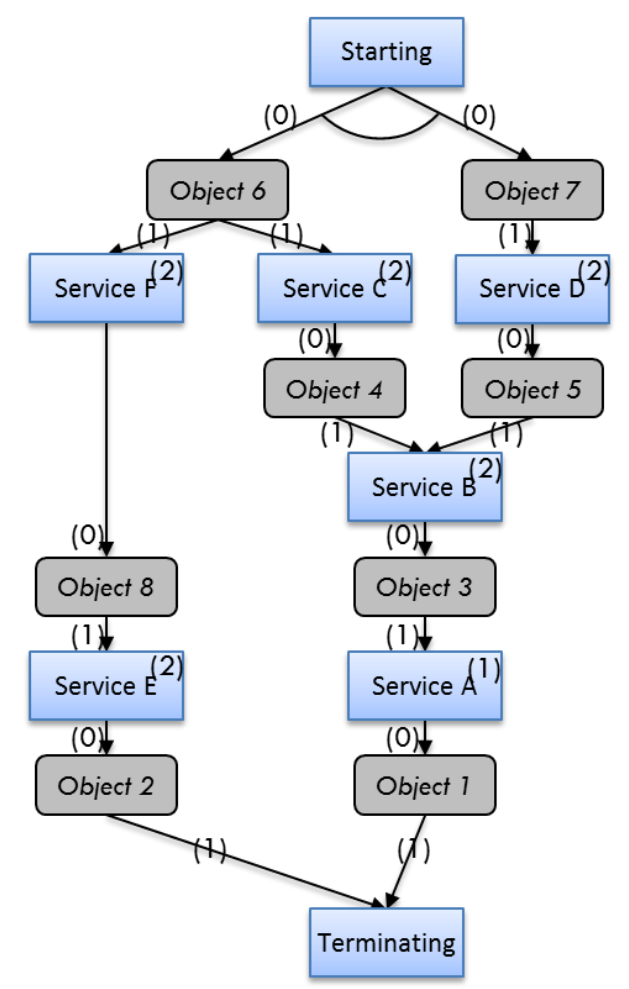

Figure 13. Resulting AND/OR graph.

\subsubsection{Minimum cost subgraph search algorithm}

Nilsson [45] introduced the AND/OR graph, or A/O graph for short, and A/O graph search problem for the first time, and since then various types of AND/OR graph search methods have been proposed.

AND/OR graph search algorithms can be categorized into explicit-graph search and implicit-graph search methods. The explicit-graph search uses an explicit data representation for the vertices and edges of an AND/OR graph, while the implicit graph search uses rules to represent them. AO* is an example of an implicit-graph search method [46].

AND/OR graph search methods can also be classified as admissible and inadmissible. While admissible algorithms guarantee that an optimal solution will be found, if one exists, inadmissible algorithms cannot guarantee that the solution found is an optimal solution. Our objective is to develop admissible search method to find the minimum cost solution graph beginning from the start vertex and leading to the terminating vertex. In the previous section, the composition network pruning and cost estimation was a kind of implicit-graph search, while the AND/OR graph search in this section is a kind of explicit-graph search, because we already have a set of candidate vertices with explicit relations between the vertices and the estimated costs.

In our algorithm, notations used in Mahanti and Bagchi [47] are adopted as follows.

- $G$ is the entire problem graph that results from the node network pruning.

- All vertices $u$ in $G$ has a finite set of successors $S(u)$.

- $\quad h^{\prime}(u)$ is an estimated cost at vertex $u$. This estimate will be used to guide the search and reduce the number of expanded vertices. 
- All edge $(u, v)$ in $G$ has a fixed cost $c(u, v)>=0$ (this is the weight on the edge).

- $P(u)$ denotes the set of predecessors of vertex $u$. For any vertex $u$ in $G, D(u)$ denotes a solution graph with root $u$.

- The subgraph of $G$ that is generated up to a certain point is called the explicit graph $G$ '.

- A cost function $h(v, G)$ on each vertex $v$ in $G$ is defined as follows:

- $h(v, G)=$ lower bound $\{h(v, D(v)) \mid D(v)$ is a solution graph with root $v$ in $G\}$, where, for a node $u$ in $D(v)$,

- $h(u, D(u))=0$, if $u$ is a terminating vertex

- $h(u, D(u))=c\left(u, u^{\prime}\right)+h\left(u^{\prime}, D\left(u^{\prime}\right)\right)$, if $u$ is an OR vertex and $u^{\prime}$ is $v^{\prime} \mathrm{s}$ immediate successor in $D(u)$

- $h(u, D(u))=\sum_{i=1}^{k}\left(c\left(u, v_{i}\right)+h\left(v_{i}, D\left(v_{i}\right)\right)\right)$, if $u$ is an AND vertex with immediate successors $v_{1}, v_{2}, \ldots, v_{k}$ in $D(u)$

Our AND/OR graph search algorithm proceeds in a top-down fashion, where each vertex expansion step is followed by a bottom-up cost revision like all AO algorithms [46]. The following pseudo code describes the procedure of our algorithm. The minimum cost solution subgraph consists of vertices labeled SOLVED after the algorithm exits.

SSCA-AND/OR-Graph-Search $(G, s) / / G$ is an AND/OR graph and $s$ is a start vertex

1 If $s$ is a terminal vertex, Then label $s$ SOLVED

2 create $G^{\prime}$ and add $s$ to $G^{\prime}$

3 While $s$ is not SOLVED

$4 \quad$ choose any unsolved successor vertex $u$ below $s$; expand $u$ generating all its successors into $S(u)$;

$5 \quad$ Add $u$ to $G$

6 for each $v \in S(u)$ not in $G^{\prime}$

$7 \quad$ add $v$ to $G$

8 If $v$ is a terminal vertex, label it SOLVED

$9 \quad$ Else compute $h(v)$

$10 \quad$ If $h(v)>h^{\prime}(v)$, BREAK

11 for each $v \in S(u)$ in $G^{\prime}$

12 recompute the costs of all predecessors in $P(v)$ assuming $h(v)=0$

13 for any $v \in S(u)$

14 If $v$ is AND vertex and $v$ has immediate predecessors other than $S(u)$,

15 Then recompute costs of all predecessors in $P(v)$ assuming $h(v)=0$

16 Clear the SOLVED labels 
17 Recompute the costs of immediate successor vertices of $s$, and label all vertices in the minimum cost subgraph as SOLVED

18 If a terminal vertex is in $G^{\prime}$, Then label $s$ SOLVED

The outer loop of the algorithm implements the top-down growth of $G^{\prime}$, while the inner loop carries out the bottom-up cost revision. The estimated costs are revised from the expanded vertex up along marked edges as well as other edges if there exists an AND vertex on the path. This revision process may change the cost of the successor vertices below the start vertex that may leads to an alternative, more promising paths.

The procedure is similar to the $\mathrm{AO}^{*}$ algorithm [ref]. The main difference is the cost revision process. Like $\mathrm{AO}^{*}$ algorithm, our algorithm also propagates its new cost back up through the graph, if the current vertex has been labeled SOLVED or its cost was just changed. In addition to that, if the current path reaches to the terminating vertex and there exist any AND vertex on the path that has another predecessor path, then our algorithm updates the cost of the vertices on the other predecessor path assuming the cost of the current AND vertex is 0. This cost revision process is necessary, because what we try to achieve is to find a minimum cost subgraph, not just a path, and there might be a shared path in a subgraph.

Mahanti and Bagchi [47] has proven that, if the cost estimation is admissible (i.e., $h^{\prime}(u) \leq h(u, G)$, $\forall u \in G$ ), then $\mathrm{AO}^{*}$ like algorithms terminate by either finding a minimum-cost solution graph rooted at $s$ or else returning $h(s)=\infty$. In our case, once the AND/OR graph is obtained from the composition network, there must be a solution because section 4.2.1.5 has already proven that our cost-estimation is admissible, i.e., $v . c_{t} \leq \delta(s, v)$.

\section{Experiment}

In this section, we compare the performance of our Service Search and Composability Analysis (SSCA) algorithm and other prominent existing AI planners in terms of effectiveness and computational efficiency. We chose OptaPlanner (V6.0.1) and BlackBox (V4.5) for the performance comparison.

The OptaPlanner is a lightweight, embeddable planning engine based on a constraint satisfaction solver [48]. Since the OptaPlanner provides various optimization heuristics and algorithms, it enables the performance comparison of our algorithm with various combinations of optimization heuristics. Throughout the experiments, Tabu Search [49], Simulated Annealing [50], and Hill Climbing [51] were used as optimization heuristics and algorithms for the constraint satisfaction.

Blackbox is a planning system that combines best features of Graphplan [31], SATPLAN [31, -52], and new randomized systematic search engines. Blackbox converts problems described in STRIPS into Boolean satisfiability (SAT) problems, and then solves the problems with existing satisfiability engines [53]. The front-end of Blackbox employs the Graphplan system [31] and for the SAT problem, Blackbox applies the local-search SAT solver such as Walksat [52] and Satz [55].

Two evaluation metrics were used in the experiments including execution time and total cost of a solution. The execution time is to measure how long each algorithm takes to find a solution. The 
time unit in this experiment is millisecond. The total cost of a solution is to measure the quality of the solution obtained. The total cost is the sum of the weights on edges in a solution.

The experiments were performed on Mac OS X version 10.9.5 with $3.5 \mathrm{GHz}$ Intel Core i7 and 8GB $1600 \mathrm{MHz}$ DDR3 RAM.

The first experiment is to analyze the correlation between the number of vertices and the performance of each planner. For this experiment, test data are randomly generated as follows.

- 50 different objects are generated for use as inputs and outputs of vertices.

- Each vertex has at least one and at most three inputs and outputs. The number of inputs and outputs are randomly selected within the restriction.

- There is no duplication between inputs and outputs of each vertex.

- For each of the test data set, user's initial condition, goal condition, and solutions are created.

We have generated total 21 different sizes of test data set by varying the size of vertices as 10,100 to 900 , and 1,000 to $10,000^{5}$. Each test data set has 4 possible solutions and 1 optimal solution. The optimal solution is a solution graph that has 3 vertices.

Table 1 and Figure 14 below show the result of the first experiment. The bold font in the table represents the best performance and the underscore represents sub-optimal result. The number in the parenthesis after the execution time shows the number of resulting vertices in the suboptimal solution. It can be seen that SSCA and Blackbox outperform CSP-based methods. Figure 15 below shows the performance comparison between SSCA and Blackbox. Blackbox produced suboptimal solutions when the number of vertices is 200, 300, and 500.

Table 1. Execution times from the experiment by varying the number of vertices

\begin{tabular}{|c|c|c|c|c|c|}
\hline $\begin{array}{ll}\# & \text { of } \\
\text { Vertices } & \end{array}$ & SSCA & Blackbox & $\begin{array}{l}\text { CSP } \\
\text { (Tabu) }\end{array}$ & $\begin{array}{l}\text { CSP } \\
(\mathrm{SA})\end{array}$ & $\begin{array}{l}\text { CSP } \\
(\mathrm{HC})\end{array}$ \\
\hline 10 & 0.002 & 0.007 & 0.037 & 0.032 & 0.027 \\
\hline 100 & 0.004 & 0.013 & 0.089 & 0.078 & 0.05 \\
\hline 200 & 0.013 & $\underline{0.02(5)}$ & 0.079 & 0.094 & 0.19 \\
\hline 300 & 0.024 & $\underline{0.026(5)}$ & 0.093 & 0.091 & 0.297 \\
\hline 400 & 0.003 & 0.004 & 0.098 & 0.134 & 0.148 \\
\hline 500 & 0.055 & $0.047(4)$ & 0.096 & 0.138 & 0.187 \\
\hline
\end{tabular}

\footnotetext{
${ }^{5}$ Ten thousand service nodes may sound unrealistically large for a services composition problem in engineering domain. However, a single service in the actual service implementation may need to be represented as multiple services in the automatic services composition problems, for example, due to flexibility to process several input formats, unit of measures, security mechanisms with different quality of service levels. This will increase the number of nodes.
} 


\begin{tabular}{|l|l|l|l|l|l|}
600 & $\mathbf{0 . 0 0 4}$ & 0.004 & 0.096 & 0.177 & 0.218 \\
\hline 700 & $\mathbf{0 . 0 0 3}$ & 0.005 & 0.109 & 0.22 & 0.231 \\
\hline 800 & $\mathbf{0 . 0 0 3}$ & 0.006 & 0.113 & 0.316 & 0.457 \\
\hline 900 & $\mathbf{0 . 0 0 4}$ & 0.004 & 0.115 & 0.107 & 0.3 \\
\hline 1000 & $\mathbf{0 . 0 0 2}$ & 0.005 & 0.115 & 0.161 & 0.215 \\
\hline 2000 & $\mathbf{0 . 0 0 3}$ & 0.006 & 0.39 & 0.952 & 0.489 \\
\hline 3000 & $\mathbf{0 . 0 0 2}$ & 0.005 & 0.415 & 0.576 & 0.409 \\
\hline 4000 & $\mathbf{0 . 0 0 4}$ & 0.007 & 0.489 & 0.445 & 0.85 \\
\hline 5000 & $\mathbf{0 . 0 0 5}$ & 0.007 & 0.603 & 1.147 & 0.966 \\
\hline 6000 & $\mathbf{0 . 0 0 5}$ & 0.008 & 0.601 & 0.34 & 1.542 \\
\hline 7000 & $\mathbf{0 . 0 0 3}$ & 0.008 & 0.917 & 1.887 & 0.434 \\
\hline 8000 & $\mathbf{0 . 0 0 6}$ & 0.01 & 1.237 & 0.926 & 1.001 \\
\hline 9000 & $\mathbf{0 . 0 0 5}$ & 0.009 & 0.659 & 0.432 & 1.355 \\
\hline 10000 & $\mathbf{0 . 0 0 6}$ & 0.009 & 0.664 & 2.351 & 0.59 \\
\hline
\end{tabular}

The execution time in both SSCA and Blackbox is quite steady regardless of the number of vertices while the execution time in CSP-based planner tends to increase in proportion to the number of vertices. That is mainly due to the fact that both the SSCA and Blackbox identify solution candidates in the forward search phase by expanding a graph from the initial states until all goal states appear. Since, in this first experiment, all the test data set has a small number of vertices in the solution graph and also each vertex has at most 3 outputs, the number of the solution candidates is small, thus those are identified quickly and that significantly reduce the entire search space. On the other hand, in the case of CSP-based planners, there is no such pruning process, thus the execution time increases as the number of vertices is increased. 


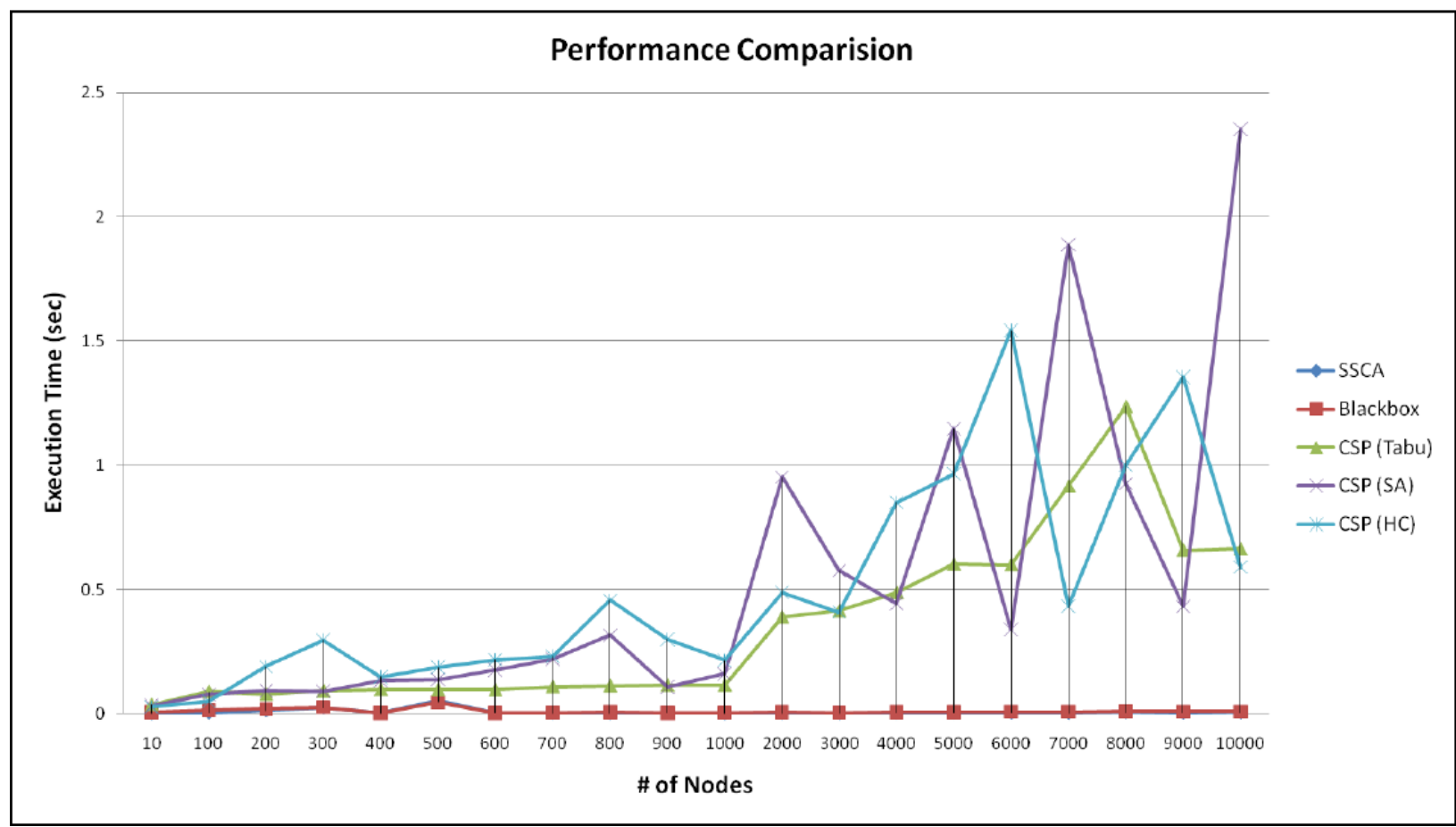

Figure 14. Performance comparison by varying the number of vertices

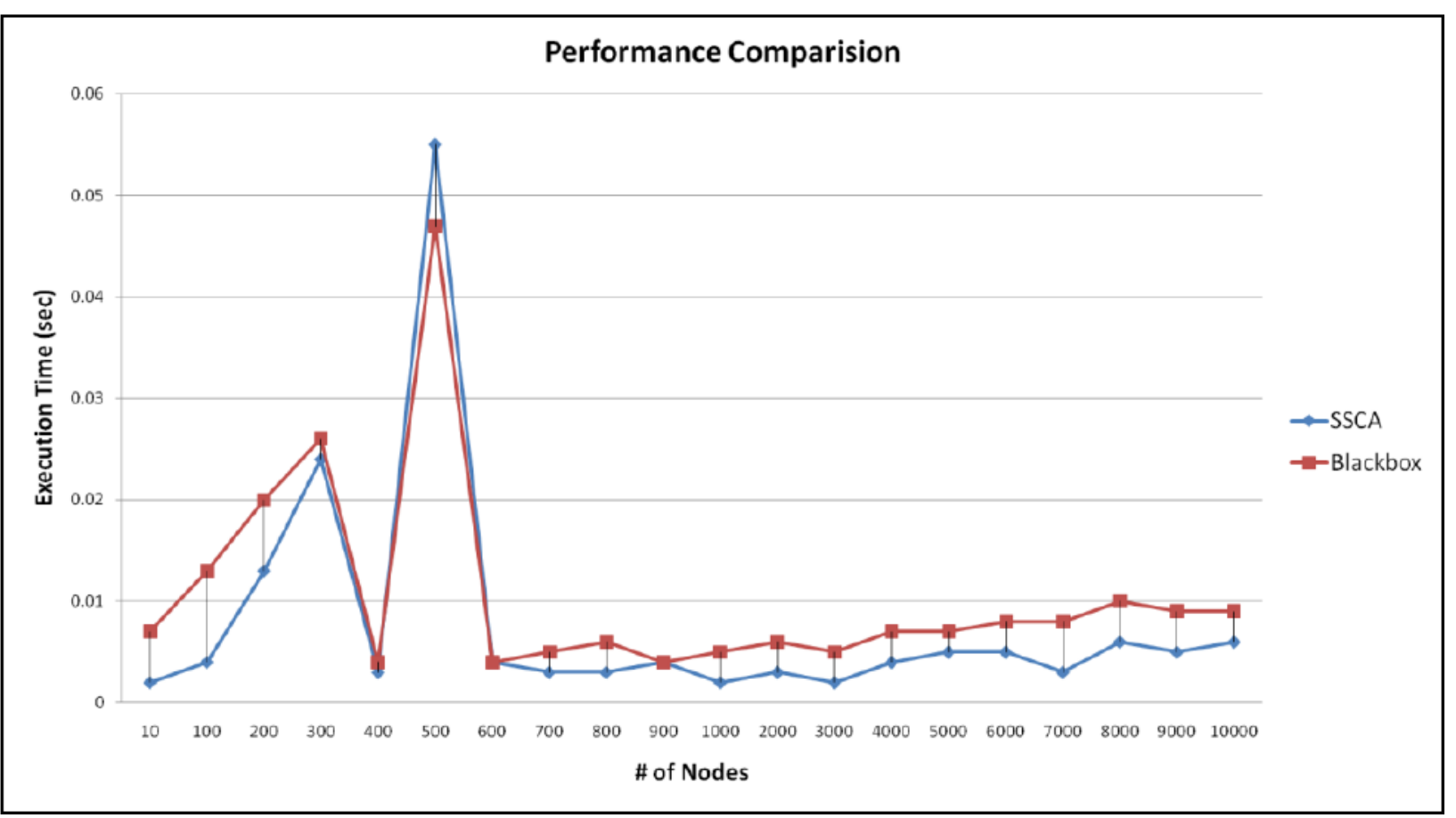

Figure 15. Performance comparison between SSCA and Blackbox

From the result of the first experiment, we observe that the number of vertices is not critical for both SSCA and Blackbox. As described in the result of the first experiment, both the SSCA and Blackbox identify solution candidates in the forward search phase by expanding a graph from the initial states until all goal states appear. If each vertex has more outgoing degree, then more vertices 
are expanded in the forward search phase and it would require longer search time. Thus, the second experiment is to analyze the correlation between the outgoing degree of vertices and the performance of each planner.

For the second experiment, test data are randomly generated as follows.

- 200 different objects are generated for use as inputs and outputs of vertices.

- 13 different test data sets are generated by varying the outgoing degree of vertices as 1,2 , $3,4,5,10,15,20,25,30,35,40$, and 45 . Outgoing degree 10 means that each vertex has 10 outputs.

- Each vertex has only one input.

- There is no duplication between inputs and outputs of each vertex. For example, if a vertex has an object as an input, then that object cannot be used as an output.

- For each of the test data set, user's initial condition, goal condition, solutions are created.

- Each test data set has a total of 1,000 vertices.

- Each test data set has 4 possible solutions and 1 optimal solution. The optimal solution is a solution graph that has 3 vertices

Table 2 and Figure 16 below show the result of the second experiment. Same as the Table 1, the bold font in the table represents the best performance and the underscore represents sub-optimal result. The number in the parenthesis after the execution time shows the number of resulting vertices in the suboptimal solution. It can be seen that SSCA outperforms the other methods. The Blackbox produced suboptimal solutions when the outgoing degree is 2 and 3. And, the Blackbox didn't work at all when the outgoing degree is greater than 3.

Table 2. Execution time from the experiment by varying the outgoing degree of vertices

\begin{tabular}{|l|l|l|l|l|l|}
\hline $\begin{array}{l}\text { Outgoing } \\
\text { Degree }\end{array}$ & SSCA & Blackbox & $\begin{array}{l}\text { CSP } \\
(\text { Tabu })\end{array}$ & $\begin{array}{l}\text { CSP } \\
(\text { SA })\end{array}$ & $\begin{array}{l}\text { CSP } \\
(\text { HC) }\end{array}$ \\
\hline 1 & $\mathbf{0 . 0 0 2}$ & 0.002 & 0.161 & 0.183 & 0.262 \\
\hline 2 & 0.024 & $\underline{\mathbf{0 . 0 1 1}(4)}$ & 0.292 & 0.173 & 0.402 \\
\hline 3 & $\mathbf{0 . 0 3 2}$ & $\underline{0.04(4)}$ & 0.396 & 0.265 & 0.139 \\
\hline 4 & $\mathbf{0 . 0 4 1}$ & - & 0.467 & 0.369 & 0.456 \\
\hline 5 & $\mathbf{0 . 0 4 3}$ & - & 0.469 & 0.104 & 1.251 \\
\hline 10 & $\mathbf{0 . 1 2 4}$ & - & 0.968 & 0.832 & 0.561 \\
\hline 15 & $\mathbf{0 . 1 7 6}$ & - & 0.895 & 1.986 & 1.725 \\
\hline 20 & $\mathbf{0 . 2 0 1}$ & - & 1.496 & 1.311 & 0.824 \\
\hline 25 & $\mathbf{0 . 2 8 5}$ & - & 2.783 & 1.703 & 0.531 \\
\hline 30 & $\mathbf{0 . 3 6 8}$ & - & 2.547 & 2.336 & 1.878 \\
\hline 35 & $\mathbf{0 . 3 8 1}$ & - & 0.576 & 3.886 & 2.285 \\
\hline 40 & $\mathbf{0 . 4 3 3}$ & - & 1.777 & 4.406 & 11.152 \\
\hline
\end{tabular}




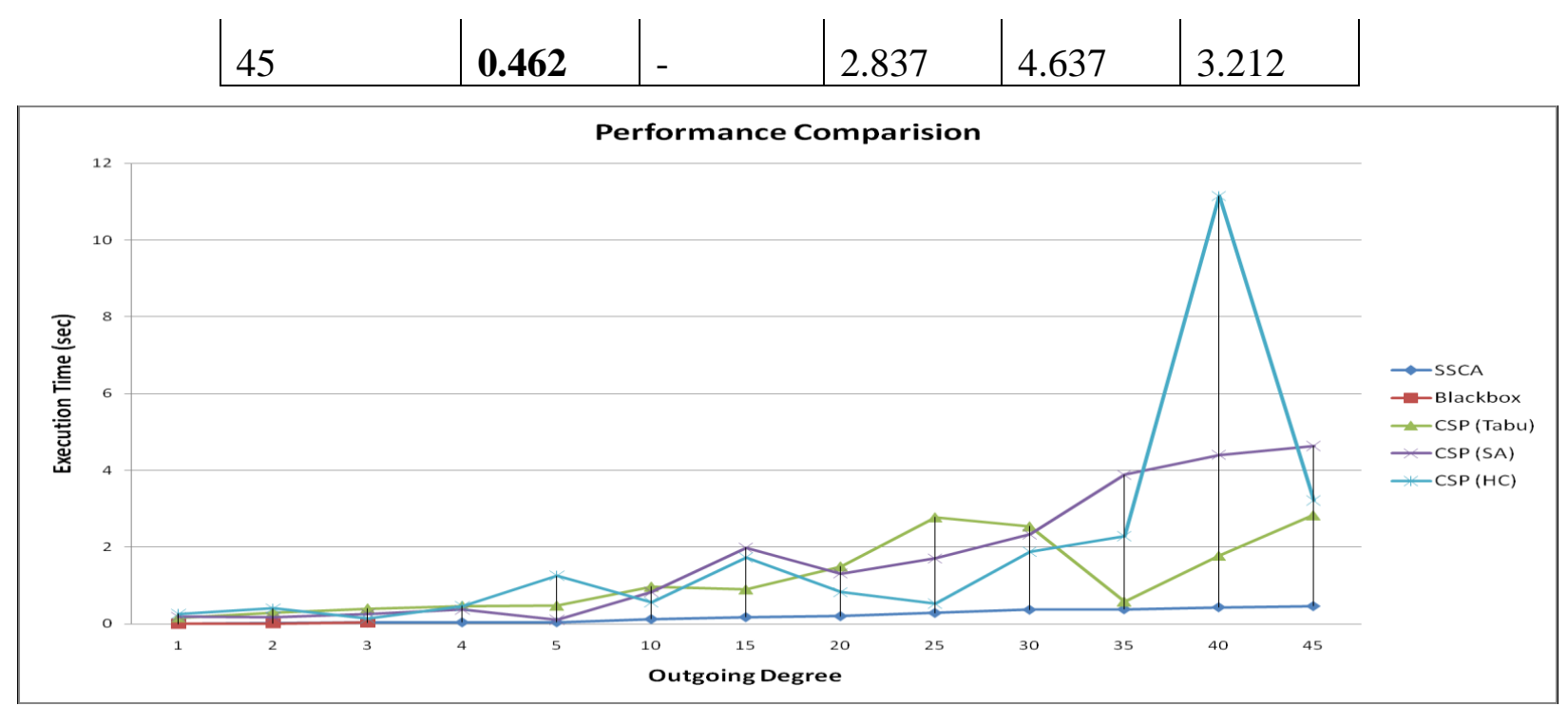

Figure 16. Performance comparison by varying the outgoing degree of vertices

\section{Discussion}

The two experiments above show that SSCA is capable of solving the service search and composition problems better than other prominent methods compared in terms of performance and scalability. Specifically, the SSCA shows a significantly better performance than those other methods when the number of vertices is large, particularly with over a thousand vertices, and the composition network is dense with tens of outgoing degrees. The Blackbox performs well but does not converge when the outgoing degree is greater than 3. The CSP-based planners require much more execution time than the SSCA. The experiments show evidences that these other methods are not as scalable as $S S C A$.

The CSP-based planners always find optimal solutions in the experiments, because the time bound was not set. However, the CSP-based planners may not find the optimal solution, if the time bound was set lesser than the execution time in the Table 1 and Table 2 . It is important to note that the computational time of SSCA did not grow exponentially when the number of vertices and the density increase; hence SSCA has a strong scalability.

\section{Conclusion}

This research developed a computer-aided services search and composition methods for an open cloud services marketplace environment. There are two main research contributions in this paper.

First, a model for formally representing a service was proposed. In this vein, our analysis of previous works in function representation theory and requirement engineering concludes that 1) both service functional and non-functional characteristics are essential to considered in services composition to achieve functional goal while maximize qualities and minimize costs; 2) functional characteristics can be device-centric or environment-centric and can be modeled as input, output, pre-condition, post-condition, and behavioral properties; and 3) non-functional characteristics can be generalized as qualities. Both behavioral properties and qualities can be commonly represented as an expression that is a fixed value, a range or equation over some parameters. 
The second research contribution is the framework for Service Search and Composability Analysis (SSCA). SSCA framework include 1) procedure to represent a services composition problem as a composition network; 2) a procedure to reduce and transform the composition network into an AND/OR graph with cost estimations; and 3) a specialized planning algorithm to find the minimum cost solution subgraph using the cost estimation as the heuristic. A proof was provided to show that the proposed cost estimation is an admissible heuristic; and hence, the SSCA heuristicbased planning algorithm will always return a minimum cost solution subgraph, if one exists, per the proof provided by a prior research. Simulation experiments were conducted; and the results affirmed the proof and demonstrated the strong scalability characteristics compared to other prominent planners.

In terms of future work, more complex cost scheme should be considered for characterizing behavioral properties and qualities such as duration, reputation, reliability, and availability and their interactions when services are composed (e.g., cross-sale and volume discount). In the current work, these characteristics are constrained to be represented as a consolidated, static cost for each service.

\section{Reference}

1. Kulvatunyou B, Ivezic N, Srinivasan, V. (2016). On architecting and composing engineering information services to enable smart manufacturing. Journal of Computing and Information Science in Engineering, 16(3), 31002.

2. Davis, J., Edgar, T., Graybill, R., Korambath, P., Schott, B., Swink, D., \& Wetzel, J. (2015). Smart manufacturing. Annual Review of Chemical and Biomolecular Engineering, 6, 141160.

3. W3C - World Wide Web Consortium (2001), Web Services Description Language (WSDL) 1.1, March 15 2001, available online at http://www.w3.org/TR/wsdl/.

4. Open API Specification version 3.0, Released July, 2017. Available online at https://www.openapis.org/.

5. Lin, S.Y., Lin, G.T., Chao, K.M. and Lo, C.C. (2012) 'A cost effective planning graph approach for large scale Web service composition', Mathematical Problems in Engineering. Article ID. 783476, pp.21.

6. Yan, Y., Chen, M., \& Yang, Y. (2012, March). Anytime QoS optimization over the PlanGraph for web service composition. In Proceedings of the 27th Annual ACM Symposium on Applied Computing (pp. 1968-1975). ACM.

7. Hatzi, O., Vrakas, D., Bassiliades, N., Anagnostopoulos, D., \& Vlahavas, I. (2013). The PORSCE II framework: Using AI planning for automated semantic web service composition. The Knowledge Engineering Review, 28(02), 137-156.

8. Nils J. Nilsson. -Artificial Intelligence: a new synthesis". Morgan Kaufmann, San Francisco, CA, USA, 2001.

9. Oh, S. C., Lee, D., \& Kumara, S. R. (2008). Effective web service composition in diverse and large-scale service networks. Services Computing, IEEE Transactions on, 1(1), 15-32. 
10. W3C - World Wide Web Consortium (2004), OWL-S: Semantic Markup for Web Services, November 22, 2004, available online at http://www.w3.org/Submission/2004/SUBM-OWLS-20041122/, accessed July 2018.

11. W3C - World Wide Web Consortium (2007), Semantic Annotations for WSDL and XML Schema, August 28, 2007, available online at http://www.w3.org/TR/sawsdl/

12. W3C - World Wide Web Consortium (2005), Web Service Modeling Ontology (WSMO), June 3, 2005, available online http://www.w3.org/Submission/WSMO/

13. Kulvatunyou, B., Wallace, E.K., Kiritsis, D., Smith, B.S., Will, C. (August 2018). The Industrial Ontologies Foundry Proof-of-Concept. In Proc. of Intl. Conf. on Advances in Production Management Systems (APMS 2018), Seoul, South Korea.

14. Kulvatunyou, B. and Ivezic, N. (June, 2002). Semantic Web for Manufacturing Web Services, International Symposium on Manufacturing and Applications (ISOMA), Orlando, Florida.

15. Jang, J., Jeong, B., Kulvatunyou, B., Chang, J., and Cho, H. Discovering and Integrating Distributed Manufacturing Services with Semantic Manufacturing Capability Profiles, International Journal of Computer-Integrated Manufacturing, 21(6), pp 631-646, 2008.

16. Barkmeyer, E. and Kulvatunyou, B. An Ontology for the e-Kanban Business Process, NIST Internal Report 7404, 2007.

17. Ameri, F., \& Dutta, D. (2006, January). An upper ontology for manufacturing service description. In ASME 2006 international design engineering technical conferences and computers and information in engineering conference (pp. 651-661). American Society of Mechanical Engineers.

18. Witherell, P., Krishnamurty, S., \& Grosse, I. R. (2007). Ontologies for supporting engineering design optimization. Journal of Computing and Information Science in Engineering, 7(2), 141150.

19. Glinz, M. (2007, October). On non-functional requirements. In Requirements Engineering Conference, 2007. RE'07. 15th IEEE International (pp. 21-26). IEEE.

20. Suzanne, R. and James, R. (1999) Mastering the Requirements Process, Addison-Wesley, Boston.

21. Sommerville, I. (2004) Software Engineering, International Computer Science Series, AddisonWesley, Boston.

22. Anton, A.I. (1997) Goal Identification and Refinement in the Specification of Information Systems. PhD Thesis, Georgia Institute of Technology.

23. Davis, A. M. (1993). Software requirements: objects, functions, and states. Prentice-Hall, Inc.

24. IEEE Computer Society. Software Engineering Standards Committee, \& IEEE-SA Standards Board. (1998). IEEE Recommended Practice for Software Requirements Specifications. Institute of Electrical and Electronics Engineers.

25. Chittaro, L., \& Kumar, A. N. (1998). Reasoning about function and its applications to engineering. Artificial intelligence in engineering, 12(4), 331-336.

26. Chakrabarti, A. (1998, July). Supporting two views of function in mechanical designs. In Proceedings 15th national conference on artificial intelligence, AAAI (Vol. 98, pp. 26-30). 
27. Chakrabarti, A., \& Bligh, T. P. (2001). A scheme for functional reasoning in conceptual design. Design Studies, 22(6), 493-517.

28. Deng, Y. M. (2002). Function and behavior representation in conceptual mechanical design. AI EDAM, 16(05), 343-362.

29. Chandrasekaran, B., \& Josephson, J. R. (2000). Function in device representation. Engineering with computers, 16(3-4), 162-177.

30. Chandrasekaran, B. (2005). Representing function: relating functional representation and functional modeling research streams. AIE EDAM, 19(02), 65-74.

31. Blum, A. L., \& Furst, M. L. (1997). Fast planning through planning graph analysis. Artificial intelligence, 90(1), 281-300

32. Kautz, H. A., \& Selman, B. (1992, August). Planning as Satisfiability. In ECAI (Vol. 92, pp. 359-363).

33. Do, M. B., \& Kambhampati, S. (2001). Planning as constraint satisfaction: Solving the planning graph by compiling it into CSP. Artificial Intelligence, 132(2), 151-182.

34. Vossen, T., Ball, M., Lotem, A., \& Nau, D. (2000). Applying integer programming to AI planning. The Knowledge Engineering Review, 15(01), 85-100.

35. Sirin, E. (2004). Automated composition of web services using AI planning techniques. (Master's thesis), Department of Computer Science, University of Maryland.

36. Hashemian, S. V., \& Mavaddat, F. (2005, January). A graph-based approach to web services composition. In Applications and the Internet, 2005. Proceedings. The 2005 Symposium on (pp. 183-189). IEEE.

37. Oh, S. C., On, B. W., Larson, E. J., \& Lee, D. (2005, March). BF*: Web services discovery and composition as graph search problem. In e-Technology, e174 Commerce and e-Service, 2005. EEE'05. Proceedings. The 2005 IEEE International Conference on (pp. 784-786). IEEE.

38. Zhang, R., Arpinar, I. B., \& AlemanMeza, B. (2003, June). Automatic Composition of Semantic Web Services. In ICWS (Vol. 3, pp. 38-41).

39. Bellman, R. (1956). On a routing problem (No. RAND-P-1000). RAND CORP SANTA MONICA CA.

40. Ford Jr, L. R. (1956). Network flow theory (No. P-923). RAND CORP SANTA MONICA CA.

41. Moore, E.F. (1957) 'The Shortest Path Through a Maze', Proceedings of an international symposium on the theory of switching, Part II, pp.285-292.

42. E. Dikjstra (1968, March). A case against the GO TO statement. ACM 11, 366-371.

43. Balakrishnan, R. and Ranganathan, K. (2012) A Textbook of Graph Theory, Springer Science \& Business Media, New York, NY.

44. M. Henzinger, S. Krinninger, and V. Loitzenbauer. 2015. Finding 2-edge and 2-vertex strongly connected components in quadratic time. In Proceedings of the 42nd International Colloquium on Automata, Languages, and Programming. 713-724. 
45. Nilsson, N. (1971) Problem Solving Methods in Artificial Intelligence, McGraw-Hill, New York, NY.

46. E. Hansen and S. Zilberstein. LAO*: A heuristic search algorithm that finds solutions with loops. Artificial Intelligence, 129:35-62, 2001.

47. A. Mahanti, A. Bagchi, AND/OR graph heuristic search methods, J. ACM 32 (1) (1985) 2851 .

48. OptaPlanner, available online at http://www.optaplanner.org//, accessed May 2018.

49. Glover, F. (1989). Tabu search-part I. ORSA Journal on computing, 1(3), 190-206.

50. Davis, L. (1987) Genetic Algorithms and Simulated Annealing, Pitman, London.

51. Davis, A.M. (1993) Software Requirements: Ob-jects, Functions, and States, Prentice Hall, Englewood Cliffs, NJ.

52. Selman, B., Kautz, H. A., \& Cohen, B. (1994, October). Noise strategies for improving local search. In AAAI (Vol. 94, pp. 337-343).

53. Fikes, R. E., \& Nilsson, N. J. (1972). STRIPS: A new approach to the application of theorem proving to problem solving. Artificial intelligence, 2(3), 189-208.

54. Selman, B., Kautz, H. A., \& Cohen, B. (1994, October). Noise strategies for improving local search. In AAAI (Vol. 94, pp. 337-343).

55. Li, C. M., \& Anbulagan, A. (1997, August). Heuristics based on unit propagation for satisfiability problems. In Proceedings of the 15th international joint conference on Artifical intelligence Volume 1 (pp. 366-371). Morgan Kaufmann Publishers Inc.. 